\title{
əImproving Nonlinear and Nonhydrostatic Ocean Lee Wave Drag Parameterizations
}

\author{
Frederick T. MAYER AND OLIVER B. Fringer \\ Bob and Norma Street Environmental Fluid Mechanics Laboratory, Civil and Environmental Engineering, \\ Stanford University, Stanford, California
}

(Manuscript received 30 March 2020, in final form 6 June 2020)

\begin{abstract}
Ocean lee waves occur on length scales that are smaller than the grid scale of global circulation models (GCMs). Therefore, such models must parameterize the drag associated with launching lee waves. This paper compares the lee wave drag predicted by existing parameterizations with the drag measured in highresolution nonhydrostatic numerical simulations of a lee wave over periodic sinusoidal bathymetry. The simulations afford a time-varying glimpse at the nonlinear and nonhydrostatic oceanic lee wave spinup process and identify a characteristic time scale to reach steady state. The maximum instantaneous lee wave drag observed during the spinup period is found to be well predicted by linear lee wave theory for all hill heights. In steady state, the simulations demonstrate the applicability of parameterizing the drag based on applying linear theory to the lowest overtopping streamline of the flow (LOTS), as is currently employed in GCMs. However, because existing parameterizations are based only on the height of the LOTS, they implicitly assume hydrostatic flow. For hills tall enough to trap water in their valleys, the simulations identify a set of nonhydrostatic processes that can result in a reduction of the lee wave drag from that given by hydrostatic parameterizations. The simulations suggest implementing a time-dependent nonhydrostatic version of the LOTS-based parameterization of lee wave drag and demonstrate the remarkable applicability of linear lee wave theory to oceanic lee waves.
\end{abstract}

\section{Introduction}

In the ocean, because lee waves occur on length scales smaller than the resolution of global circulation models (GCMs), the lee wave drag must be parameterized. Existing parameterizations are based on steady-state solutions for the flow above "linear-height" hills, wherein the height of the hill is much smaller than the wavelength of the wave (Bell 1975b; Gill 1982). Most common among the unresolved bathymetric features of the deep ocean are the abyssal hills which are statistically homogeneous on a regional scale and thus submit well to a spectral model (Goff and Jordan 1988). This allows for calculation of the lee wave drag with the spectral linear theory of Bell $(1975 a, b)$. However, because a significant portion of the abyssal hills have nonlinear heights (Nikurashin et al.

¿ Denotes content that is immediately available upon publication as open access.

Corresponding author: Frederick T. Mayer, fmayer@ stanford.edu
2014), spectral linear theory is not formally valid in these regions.

At the bottom of the ocean, where the background horizontal velocity $U$ and buoyancy frequency $N$ are nearly constant with height, lee wave dynamics can be described in terms of the lee wave Froude number $J=$ $N h_{0} / U$ and the nonhydrostatic parameter $\epsilon=U k / N$, where $h_{0}$ and $k$ are the hill height and wavenumber (Mayer and Fringer 2017). Using the lee wave Froude number $J$, nonlinear lee waves can be separated into two regimes: subcritical, $J=N h_{0} / U<J_{c}$, and supercritical, $J=N h_{0} / U \geq J_{c}$, where $J_{c}=O(1)$ is the critical Froude number. The supercritical regime is characterized by dramatic deviations of the form drag from the predictions of linear theory. Above isolated bathymetry, the flow on the downslope side of the isolated hill displays a standing hydraulic jump-like feature called a "downslope windstorm," with an associated pressure anomaly resulting in as much as an eightfold amplification of the steady-state drag above the linear prediction (Peltier and Clark 1979; Pierrehumbert 1987). However, for supercritical lee waves above periodic bathymetry, such as the abyssal hills, the upslope of the next hill inhibits 
the formation of the downslope windstorm, and the flow instead displays a process called "blocking," wherein the lowest parcels of water become trapped in the valleys (e.g., Welch et al. 2001; Nikurashin and Ferrari 2010; Winters 2016). In the presence of blocking, some water passes over the hills and generates lee wave energy. In steady state, this overtopping fluid travels along a streamline that is different from the bathymetry. The resulting wave is therefore identical to a subcritical lee wave generated by the lowest overtopping streamline, which we will refer to as the LOTS. High-resolution nonlinear numerical simulations of atmospheric lee waves demonstrate that the supercritical lee wave drag scales with the trough-to-crest height of the LOTS, which is observed to be $O(U / N)$ (Stein 1992; Welch et al. 2001; Eckermann et al. 2010). Thus, atmospheric wave drag parameterizations posit that the wave drag over supercritical height hills is given by substituting an effective Froude number $J_{\text {eff }} \approx 1$ for $J$ in linear theory (Palmer et al. 1986; Pierrehumbert 1987; Lott and Miller 1997; Garner 2005). We will refer to this approach as saturation theory.

As a package, the spectral model of the abyssal hills combines with saturation theory to permit computationally cheap calculations of the lee wave drag over arbitrary height, subgrid-scale abyssal hills in ocean models. This is the approach employed in recent studies of GCMs (Nikurashin and Ferrari 2011; Scott et al. 2011; Naveira Garabato et al. 2013; Melet et al. 2015; Trossman et al. 2013, 2015, 2016; Wright et al. 2014; Yang et al. 2018, 2019). These studies conclude that globally, lee wave drag constitutes between 0.2 and 0.75 TW of work on the ocean, meaning lee waves could be of principle importance for balancing the $O(1) \mathrm{TW}$ of wind work at the ocean surface (Ferrari and Wunsch 2009). However, direct observations of lee waves in supercritical regions such as the Drake Passage (Cusack et al. 2017) and Kerguelen Plateau (Waterman et al. 2013) display dissipation rates one order of magnitude smaller than predicted by combining saturation theory with an energy-dissipation parameterization (St. Laurent et al. 2002). To account for this discrepancy, recent papers have implicated the interaction of geostrophic and tidal currents (Shakespeare 2020) and the absorption of wave energy aloft via conservation of wave action in vertically sheared flow (Kunze and Lien 2019). In their introduction, Kunze and Lien (2019) offer a handful of additional hypotheses, including measurement and instrument error, under sampling, wave-wave interaction, a narrow radiating bandwidth, and, finally, that lee wave saturation is incompletely parameterized.

Saturation theory, as presented above, is based on lee waves of atmospheric scale, where the forcing is hydrostatic.
Assuming hydrostatic forcing implies that the wave drag is only a function of hill height, or nondimensionally, of $J$, and not also a function of hill length (nondimensionally $\epsilon$ ) (Pierrehumbert 1987). However, in the $J-\epsilon$ regime appropriate to lee waves over abyssal hills in the ocean, nonhydrostatic effects are important, even for subcritical height bathymetry (Mayer and Fringer 2019, manuscript submitted to Ocean Modell.). Nikurashin and Ferrari (2010) and Nikurashin et al. (2014) are an exception to the existing lee wave literature in that their simulations employ high-resolution nonhydrostatic models and capture a $J-\epsilon$ regime appropriate for abyssal hill lee waves. However, both studies derive their prediction for $J_{\text {eff }}$ without varying the horizontal component of the generating bathymetry. Thus, although their simulations incorporate nonhydrostatic processes, using their result for $J_{\text {eff }}$ to parameterize the lee wave drag above arbitrary bathymetry is still implicitly hydrostatic because it assumes that the drag is independent of hill length.

Additionally, existing saturation based parameterizations assume both that the lee wave has reached a steady state and that the momentum radiating through the wave is deposited within the first wavelength above the bathymetry. However, for a linear lee wave in regions with negligible rotational effects, the drag is an intrinsically unsteady feature that propagates vertically with the lee wave front, such that the drag only acts on the bottom currents during the earliest period of lee wave generation (Pedlosky 2003). This vertically propagating lee wave drag should also exist in the supercritical regime because supercritical hills still launch subcritical lee waves. Although rotation complicates this by permitting lee wave-inertial oscillation interaction (Nikurashin and Ferrari 2010), the assumption of steadystate lee waves depositing their momentum within the first wavelength above the bathymetry is likely not justified everywhere in the ocean.

This paper offers a process study of an idealized lee wave to illuminate the consequence of ignoring nonhydrostatic and unsteady effects when parameterizing oceanic lee wave drag. The results are based on highresolution, nonhydrostatic simulations of lee waves above sinusoidal hills spanning the $J-\epsilon$ range of oceanic flow over abyssal hills. The paper is organized as follows: in section 2 we review the linear theory. In section 3 we describe the numerical model and discuss methods for measuring the lee wave drag. In section 4 we analyze the temporal evolution of a subset of subcritical and supercritical simulations. In section 5 we evaluate the steady-state drag and LOTS measured in all of the simulations. And in section 6 we conclude with recommendations for nonhydrostatic and time-dependent corrections to saturation theory. 


\section{Linear theory and parameter space}

As in Mayer and Fringer (2017), we assume a nonrotating ocean with a free-slip bottom boundary condition and an infinite depth. Although rotation is important at the scale of abyssal hills $[O(1-10) \mathrm{km}]$ in much of the ocean, where its primary contribution is to enforce a lower bound on the radiating bandwidth of lee waves according to $f<U k<N$, the following theory and simulations are greatly simplified by omitting it. We discuss the ramifications of ignoring rotation in the conclusions. With the above assumptions, the lee wave is uniquely characterized by $J=N h_{0} / U$ and $\epsilon=U k / N$. Mayer and Fringer (2017) demonstrate that $J=N h_{0} / U$ is the lee wave Froude number formed by the ratio of perturbation advection within the lee wave to the group velocity of the lee wave. The second dimensionless number, $\epsilon=U k / N$, can be cast as a ratio of the wavelength of the lee wave in the direction of the wave vector, $\lambda_{\text {lee }}=2 \pi U / N$, to the horizontal wavelength of the bathymetry, $L_{\text {hill }}=2 \pi / k$. In terms of $\epsilon$, the vertical wavenumber of the lee wave is given by

$$
m=\frac{N}{U}\left(1-\epsilon^{2}\right)^{1 / 2}
$$

With increasing $\epsilon$ (increasing nonhydrostasy), the lee wave wavenumber vector points increasingly downstream. When $\epsilon>1$, the wavenumber of the hill is larger than the lee wave wavenumber, implying an imaginary vertical wavenumber and thus a decaying, or evanescent, lee wave.

Abyssal hills are periodic and quasi-anisotropic in nature, with horizontal aspect ratios of between 1:3 and 1:8 (Goff and Arbic 2010; Fig. 1), such that they appear as ridge-like corrugations on the ocean floor. As an idealization, we consider the simple sinusoidal bathymetry

$$
h(x)=\frac{1}{2} h_{0} \sin (k x) \text {. }
$$

The factor of $1 / 2$ is used so that $h_{0}$ is the trough to crest height of the hill. This convention follows Lilly and Klemp (1979) and Welch et al. (2001), but differs from Nikurashin and Ferrari (2010), where the authors omit the factor of $1 / 2$. Hence, the height scale in Nikurashin and Ferrari (2010) relates to $h_{0}$ as $h_{\mathrm{NF}}=h_{0} / 2$. Using Eq. (2), Mayer and Fringer (2019, manuscript submitted to Ocean Modell.) show that the dimensionless steadystate nonhydrostatic linear form drag per wavelength is given by

$$
\frac{F_{\operatorname{lin}}}{\rho_{0} U^{3} N^{-1}}= \begin{cases}\frac{\pi}{4} J^{2}\left(1-\epsilon^{2}\right)^{1 / 2}, & \epsilon<1, \\ 0, & \epsilon \geq 1 .\end{cases}
$$

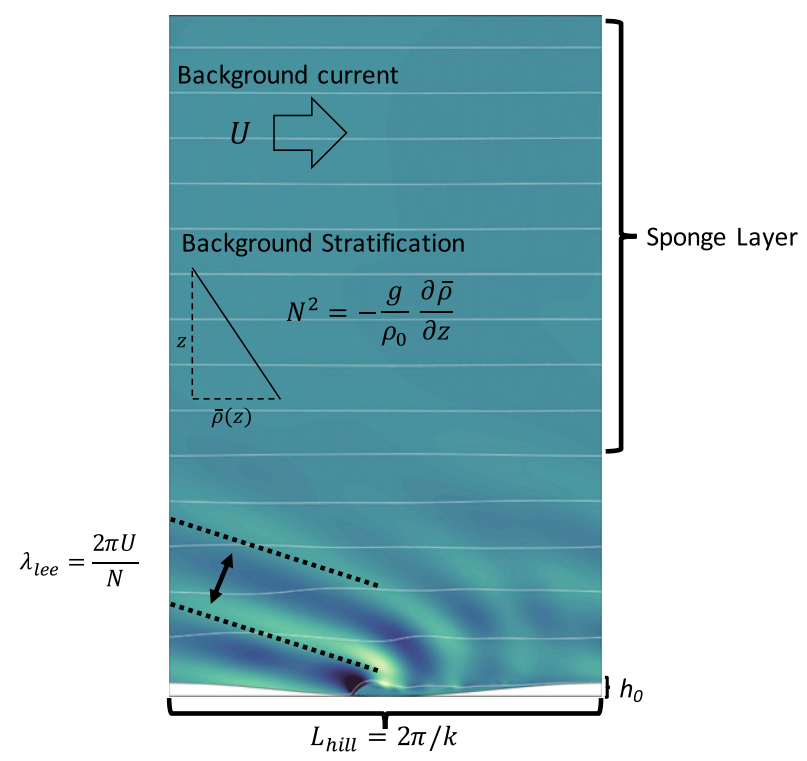

FIG. 1. A lee wave generated over sinusoidal bathymetry of length $L_{\text {hill }}$ and height $h_{0}$ resulting from uniform background flow with horizontal velocity $U$ and buoyancy frequency $N^{2}=g / \rho_{0}(\partial \bar{\rho} / \partial z)$, where $g$ is the gravitational acceleration, $\bar{\rho}$ is the background density of the fluid, and $\rho_{0}$ is the reference density. The color contours and white lines are the instantaneous vertical velocity and simulated streamlines at time $t=T_{\mathrm{ex}}=L_{\mathrm{hill}} / U$, taken from one of the simulations reported in this paper $(J=1.2$ and $\epsilon=0.16)$. The characteristic wavelength between lines of constant phase is $\lambda_{\text {lee }}=2 \pi U / N$, as sketched with dotted black lines. The color map is "deep" from Thyng et al. (2016).

Equation (3) indicates that the nondimensional drag on the background current associated with generating lee waves grows in proportion to $J^{2}$, but decreases with decreasing hill length (increasing $\epsilon$ ) until the evanescent boundary $L_{\text {hill }}=\lambda_{\text {lee }}$, beyond which the steady-state drag vanishes.

Equation (3) affords a number of important limits and extrapolations. The hydrostatic limit of Eq. (3) is given by setting $\epsilon=0$,

$$
\frac{F_{\mathrm{hs}}}{\rho_{0} U^{3} N^{-1}}=\frac{\pi}{4} J^{2},
$$

and represents the largest magnitude drag that can result from a lee wave over a hill with crest to trough height $h_{0}$. From this expression, a scale for the computed drag in what follows is given by

$$
F_{\text {sat }}=\rho_{0} U^{3} N^{-1} .
$$

Finally, the prediction for drag offered by saturation theory can also be stated using Eq. (3), as in Yang et al. (2018),

$$
F_{\text {Yang }}=F_{\text {lin }} L^{2},
$$


where

$$
L= \begin{cases}1, & J<J_{c}, \\ J_{\mathrm{eff}} / J, & J \geq J_{c},\end{cases}
$$

$J_{c}=O(1)$ is an empirically determined critical Froude number above which the drag saturates, and $J_{\text {eff }}$ is the Froude number of the resulting effective bathymetry. It is generally assumed that $J_{\text {eff }}=J_{c}$. As a canonical example, in their nonhydrostatic simulations of lee waves above $\epsilon=0.31$ sinusoidal hills, Nikurashin and Ferrari (2010) find that saturation occurs when $N h_{\mathrm{NF}} / U=0.7$. Recalling that $h_{\mathrm{NF}}=h_{0} / 2$, this implies a critical Froude number $J_{\mathrm{NF}}=1.4$.

All existing parameterizations assume a steady process in which the lee wave extracts momentum continuously from the currents within one lee wave wavelength $(\approx 500 \mathrm{~m})$ of the bottom. However, irrotational linear theory (e.g., Pedlosky 2003) suggests that the lee wave drag only acts on the background current in the vicinity of the wave front, which propagates with vertical group velocity

$$
C_{z}=\epsilon U\left(1-\epsilon^{2}\right)^{1 / 2}
$$

This picture is complicated by rotation, where the more hydrostatic hills, for which $U k \rightarrow f$, result in an infinite vertical wavenumber, a vanishing vertical group velocity, and thus an evanescent response (Klymak 2018; Kunze and Lien 2019). Nevertheless, the general observation that the lee wave drag is felt only within the vicinity of the vertically propagating wave front remains valid. Presumably, the same temporal effect is present in nonlinear lee waves because, as posited by saturation theory, there is still a subcritical lee wave generated by the LOTS, and thus still a wave front where the lee wave drag acts.

The theory presented in this section suggests that lee waves above nonlinear and nonhydrostatic bathymetry should exhibit nonlinear, nonhydrostatic, and timedependent behavior. However, at present, saturation theory offers a hydrostatic and steady-state parameterization for the lee wave drag. To quantify significance of omitting nonhydrostatic and time-dependent effects, we turn to numerical simulations of the idealized oceanic lee wave.

\section{Numerical model}

\section{a. Model setup}

Our simulations employ the nonhydrostatic Navier Stokes solver SUNTANS (Fringer et al. 2006). The setup, sketched in Fig. 1, is identical to that in Mayer and
Fringer (2019, manuscript submitted to Ocean Modell.), with a uniform buoyancy frequency $N=0.002 \mathrm{~s}^{-1}$ and constant volume-averaged horizontal velocity $U=0.2 \mathrm{~m} \mathrm{~s}^{-1}$ such that the lee wave wavelength is $\lambda_{\text {lee }}=2 \pi U / N=628 \mathrm{~m}$. Because we are primarily interested in lee waves in the deep ocean, where the free surface plays a negligible role, the domain in all simulations is $7 \mathrm{~km}$ deep and employs a sponge layer throughout the upper $5 \mathrm{~km}$. The vertical grid spacing is a constant $\Delta z=5 \mathrm{~m}$ beneath the sponge layer (the bottom $2 \mathrm{~km}$ of the domain), and stretches linearly within the sponge layer until $\Delta z=$ $300 \mathrm{~m}$ at the surface. This is identical to the vertical grid used in Nikurashin and Ferrari (2010). The simulations begin at rest and are spun up with a forcing scheme that ensures a constant volume-averaged streamwise velocity $U$ (Nelson and Fringer 2017). As described in Mayer and Fringer (2019, manuscript submitted to Ocean Modell.), the kinematic viscosity is a constant $\nu=$ $0.01 \mathrm{~m}^{2} \mathrm{~s}^{-1}$, and no explicit turbulence model is employed. Shakespeare and Hogg (2017) suggest that this value for $\nu$ is small enough to produce an effectively inviscid linear lee wave simulation (see their Fig. 3.c). Tests of our model setup with smaller viscosity (not shown) display little dependence on the Reynolds number, defined by $\operatorname{Re}=U /(\nu k)$.

Whereas in Mayer and Fringer (2019, manuscript submitted to Ocean Modell.), we only varied the length of the sinusoidal hill [Eq. (2)], in this paper we now vary both the height and length over the set $h_{0}=$ [2 to $\left.200 \mathrm{~m}\right]$ and $L_{\text {hill }}=[4 \mathrm{~km}$ to $800 \mathrm{~m}]$. This samples the parameter space $J=[0.02$ to 2$]$ and $\epsilon=[0.16$ to 0.8$]$, which is characteristic of lee waves over abyssal hills (Goff and Arbic 2010; Nikurashin and Ferrari 2011). Anticipating that nonlinear-height lee waves might take longer to reach steady state, each simulation in this paper is run for 20 excitation periods, $T_{\mathrm{ex}}=L_{\mathrm{hill}} / U$. Additionally, all simulations are nonhydrostatic with a constant horizontal resolution of $\Delta x=10 \mathrm{~m}$. This avoids both the unphysical overprediction of the wave drag caused by using a hydrostatic model with nonhydrostaticlength bathymetry as well as the underprediction in wave drag caused by poorly resolving the bathymetry (Mayer and Fringer 2019, manuscript submitted to Ocean Modell.).

\section{b. Computing the lee wave drag}

This study employs two methods to compute the simulated lee wave drag. The first uses the simulated pressure $p$ at the bottom $[z=h(x)]$ and the analytical bathymetry $h(x)$ to directly compute the form drag as

$$
F_{\text {form }}=\int_{0}^{\text {Lhill }} p[x, z=h(x)] \frac{\partial h}{\partial x} d x .
$$


A second measurement of the lee wave drag is provided by integrating the vertical momentum flux through a horizontal plane above the bathymetry. Unless otherwise noted in what follows, we choose this plane to be a height $z_{t}=15 \mathrm{~m}$ above the crest of the hill, and define

$$
F_{\text {flux }}=\int_{0}^{\text {Lhill }} \rho_{0} u\left(x, z_{t}\right) w\left(x, z_{t}\right) d x
$$

For a steady inviscid lee wave over a linear hill, $F_{\text {form }}=-F_{\text {flux }}$ analytically. Viscous effects are negligible in the ocean, and still small for $z_{t} \ll \lambda_{\text {lee }}$ even with the relatively large viscosity in our model (Shakespeare and Hogg 2017).

The relationship between the form drag and momentum flux is demonstrated by the governing areaintegrated horizontal momentum equation, which is given by

$$
\frac{d M}{d t}=-F_{\text {flux }}-F_{\text {form }}
$$

where

$$
M=\rho_{0} \int_{A} u d A
$$

is the area-integrated momentum per unit span. In equilibrium $d M / d t=0$, implying $F_{\text {flux }}=-F_{\text {form }}$. However, the time-integrated momentum equation produces nonzero $M$ when unsteadiness and nonlinearities lead to imbalances between $F_{\text {flux }}$ and $-F_{\text {form }}$, as discussed in what follows.

\section{Unsteady behavior of lee waves}

\section{a. Subcritical, weakly nonlinear case $[J=O(0.1)]$}

Before interrogating the supercritical simulations, in this section we consider a simulation over a weakly nonlinear but still subcritical height hill. For this purpose, the 4-km-long and 60-m-tall hill $(\epsilon=0.16, J=0.6)$ is a good example. Snapshots of the nondimensional vorticity $\left[\omega^{*}=\omega /(J N)\right]$ and streamlines every half $T_{\mathrm{ex}}$ are shown in Fig. 2. Note that the wave front travels upward with group velocity, $C_{z}=\epsilon U\left(1-\epsilon^{2}\right)^{1 / 2}$ [Eq. (8)]. For this hydrostatic hill, the nonhydrostatic effect is negligible since $\left(1-\epsilon^{2}\right)^{1 / 2}=0.987$. Hence, $C_{z} \approx \epsilon U=$ $\lambda_{\text {lee }} / T_{\text {ex }}$ such that after one excitation period, the wave front has traveled approximately one wavelength into the water column. After two excitation periods, the wave is fully established within the domain shown, and little changes over the following half $T_{\mathrm{ex}}$.

The lowest parcel of water in this weakly nonlinear simulation struggles to flow over the hill during spinup,
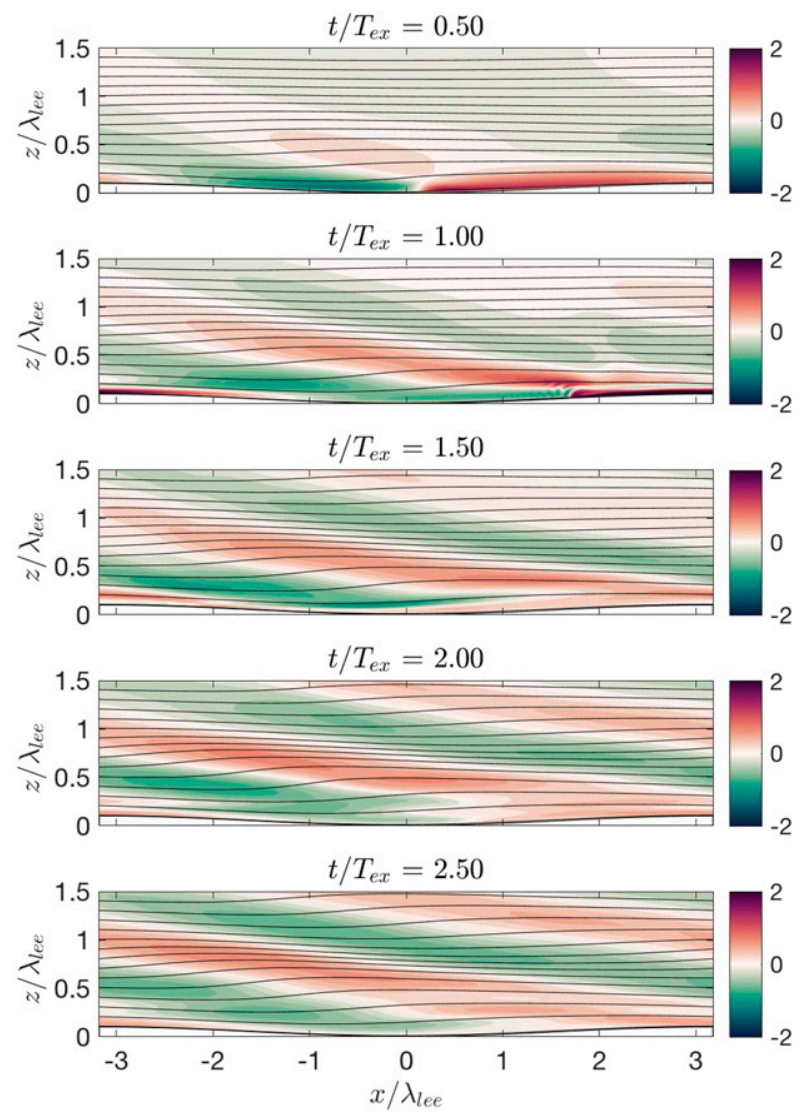

FIG. 2. Nondimensional vorticity $\left[\omega^{*}=\omega /(J N)\right]$ and streamlines at half $T_{\mathrm{ex}}$ intervals for the simulation with $J=0.6$ and $\epsilon=0.16$ $\left(h_{0}=60 \mathrm{~m}, L_{\text {hill }}=4 \mathrm{~km}\right)$. The color map is "curl" from Thyng et al. (2016).

such that at $t=T_{\mathrm{ex}} / 2$, in Fig. 2, there is a small jump in vorticity halfway up the hill. By the next snapshot, at $t=T_{\mathrm{ex}}$, this bottom water has traveled over the hill and no longer presents an obstacle. Nevertheless, the sharp gradients in vorticity lead to mixing and a reduction of the buoyancy frequency near the bottom. Figure 3 shows the horizontally averaged instantaneous buoyancy frequency $\langle N\rangle$ for this simulation at times corresponding to those in Fig. 2, as well as later times, where we define $\langle N\rangle$ as

$$
\langle N\rangle=\frac{1}{L_{\text {hill }}} \int_{0}^{L_{\text {hill }}}\left(-\frac{g}{\rho_{0}} \frac{\partial \rho}{\partial z}\right)^{1 / 2} d x .
$$

In Fig. 3, the decay of the buoyancy frequency near the bottom is significant. By $t=T_{\mathrm{ex}},\langle N\rangle$ at the bottom of the valley is roughly $25 \%$ smaller than the background $N$, and it continues to decay throughout the simulation. However, the decay remains confined to a region $\delta=$ $U / N$ above the hill (indicated by the dashed horizontal line in Fig. 3). A similar bottom-confined decay of 


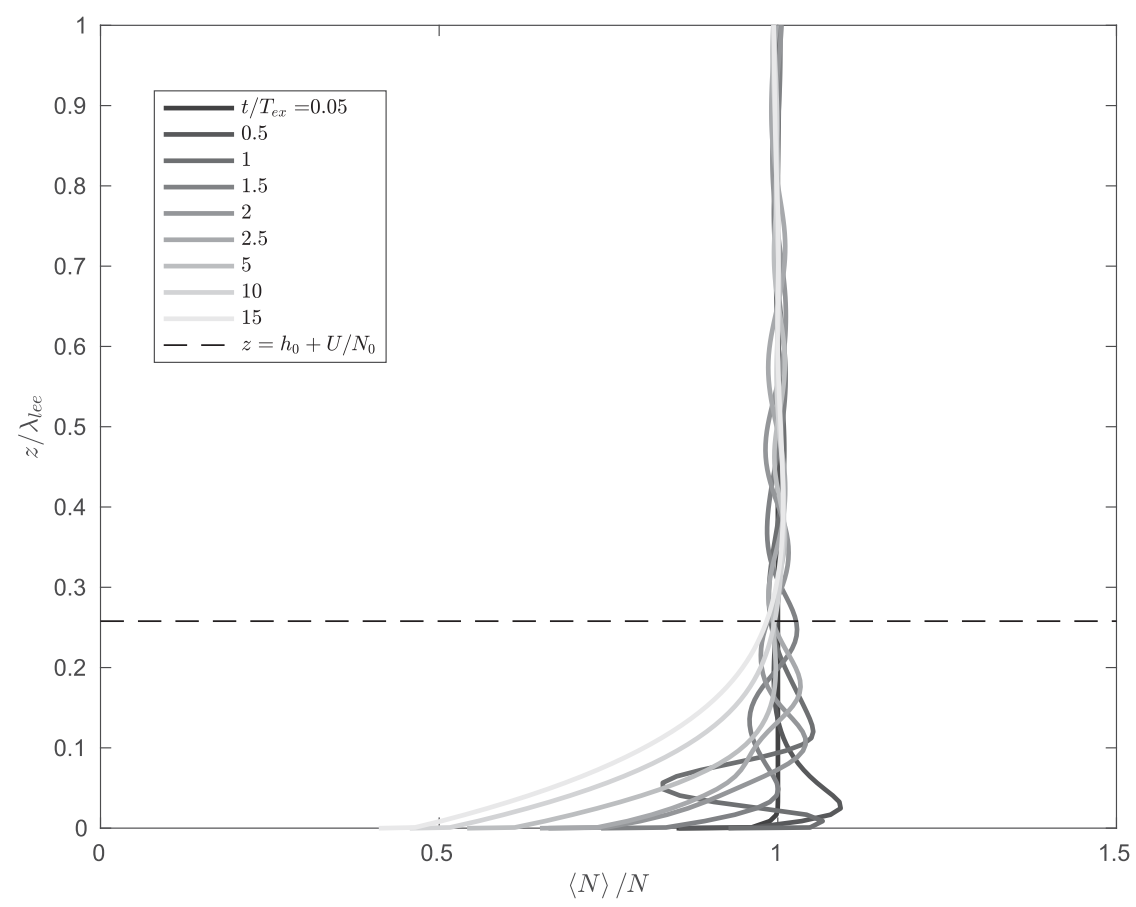

FIG. 3. Horizontally averaged local buoyancy frequency $\langle N\rangle$ [Eq. (13)] as a function of height above the valley bottom for the simulation shown in Fig. 2. Each line corresponds to a moment in the simulation, as indicated in the legend. The horizontal dashed line is at a height $z=h_{0}+U / N\left[z / \lambda_{\text {lee }}=(J+1) / 2 \pi\right]$ above the valley bottom.

buoyancy frequency was observed in the lee wave simulations of Klymak (2018), where it was likewise attributed to the spinup of the system from rest. There is reason to believe that the phenomenon exists in nature. For example, observations in locations ripe for lee waves such as the Antarctic Circumpolar Current north of the Kerguelen Plateau (Waterman et al. 2013) and the Hoyt Hills region of the Gulf current (Zheng et al. 2012) indicate bottom layers with thickness approximately equal to $U / N$. Note that this decay of near-bottom buoyancy frequency is only an issue for periodic bathymetry. For an isolated hill, the mixing during spinup would momentarily reduce the stratification above the hill, but not that of upstream fluid that flows over the hill at later times. Hence the steady-state dynamics of a lee wave above a given hill are only sensitive to the local decay of buoyancy frequency during spinup when there are other hills upstream to do the initial mixing.

To approximate the effect of a decayed bottom buoyancy frequency on the lee wave above periodic bathymetry, we define an adjusted bottom-layer buoyancy frequency by averaging $\langle N\rangle$ from $z=0$ to $z=h_{0}+$ $U / N$ with

$$
N_{\text {adj }}=\frac{1}{h_{0}+U / N} \int_{0}^{h_{0}+U / N}\langle N\rangle d z,
$$

where $z=0$ is taken at the bottom of the valley. Inspection of Eq. (4) shows that the hydrostatic wave drag is proportional to the buoyancy frequency, $F_{\operatorname{lin}} \propto N$. Thus a reduction in the buoyancy frequency over a layer $\delta$ above the bathymetry should result in smaller form drag, which we can approximate by substitution of $N_{\text {adj }}$ for $N$ in Eq. (3), giving

$$
F_{\text {adj }}=\frac{N_{\text {adj }}}{N} F_{\text {lin }} .
$$

Note that this expression ignores the nonhydrostatic effect of $N_{\text {adj }}$ on the vertical wavenumber [Eq. (1)]. We found that incorporating an adjusted vertical wavenumber $m_{\text {adj }}$ produced unphysical predictions, especially above the more nonhydrostatic hills. Above these narrow hills, $N_{\text {adj }}$ implies imaginary $m_{\text {adj }}$ and evanescent waves (since $U k>N_{\text {adj }}$ ), even though the disturbance generates a propagating wave in the region above the decayed buoyancy. In Fig. 4, we compare $F_{\text {adj }}$ to the computed form drag [Eq. (9)] and vertical momentum flux [Eq. (10)] in the subcritical simulation shown in Fig. 2. The adjusted linear theory accurately predicts the general evolution of the computed drag over the course of the simulation. As demonstrated in the following section, the adjusted linear theory works equally well during steady state (see Figs. 10, 11). Hence, for 


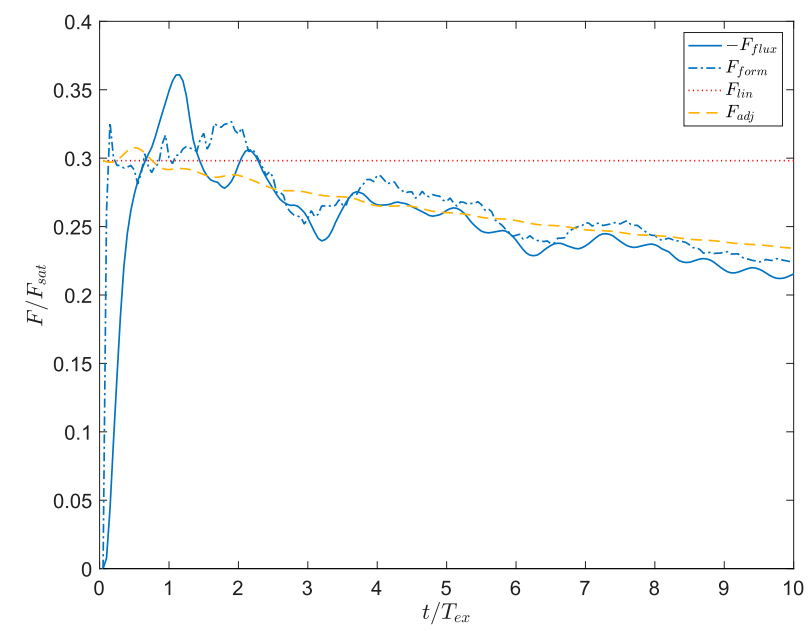

FIG. 4. Computed form drag [dot-dashed line, Eq. (9)] and vertical momentum flux through a plane $15 \mathrm{~m}$ above the crest of the hill [solid line, Eq. (10)] as a function of time for the simulation in Fig. 2. Also shown are the predictions of linear theory using the background buoyancy frequency [dotted line, Eq. (3)] and the adjusted buoyancy frequency [dashed line, Eq. (15)].

subcritical simulations, we should expect a smaller drag than predicted by linear theory, $F_{\text {lin }}$ [Eq. (3)], due to the decay of buoyancy in the lowest layers. Additionally, in regions where rotation is important, the decay in buoyancy frequency narrows the $N-f$ frequency band for propagating lee waves. For a field of abyssal hills with varying wavenumber, the decay of near-bottom buoyancy frequency thus has the consequence of further reducing the total lee wave drag (Kunze and Lien 2019).

The subcritical example also affords an opportunity to study the progression of the wave front as it propagates away from the hill. In Fig. 5, we display $F_{\text {flux }}$ [Eq. (10)] as a function of height above the bottom at different points in time. Note that the wave front has a vertical extent of roughly one lee wave wavelength, $\lambda_{\text {front }} \approx \lambda_{\text {lee }}$, and propagates vertically at a rate of approximately one $\lambda_{\text {lee }}$ for each $T_{\text {ex }}$, as predicted by hydrostatic linear theory. Also note that after the wave front has reached the sponge layer, the momentum flux is nearly constant with height, with a slight decay with distance from the bottom as predicted by viscous effects (Shakespeare and Hogg 2017). The nondivergent momentum flux confirms the hypothesis of Pedlosky (2003) that in the subcritical regime after spinup, the lee wave drag is not felt by the lowest water, where the momentum flux is nondivergent, but aloft in the vicinity of the propagating wave front. From the perspective of a GCM, this supports the notion that lee wave drag parameterizations could account for unsteadiness by appropriately positioning the

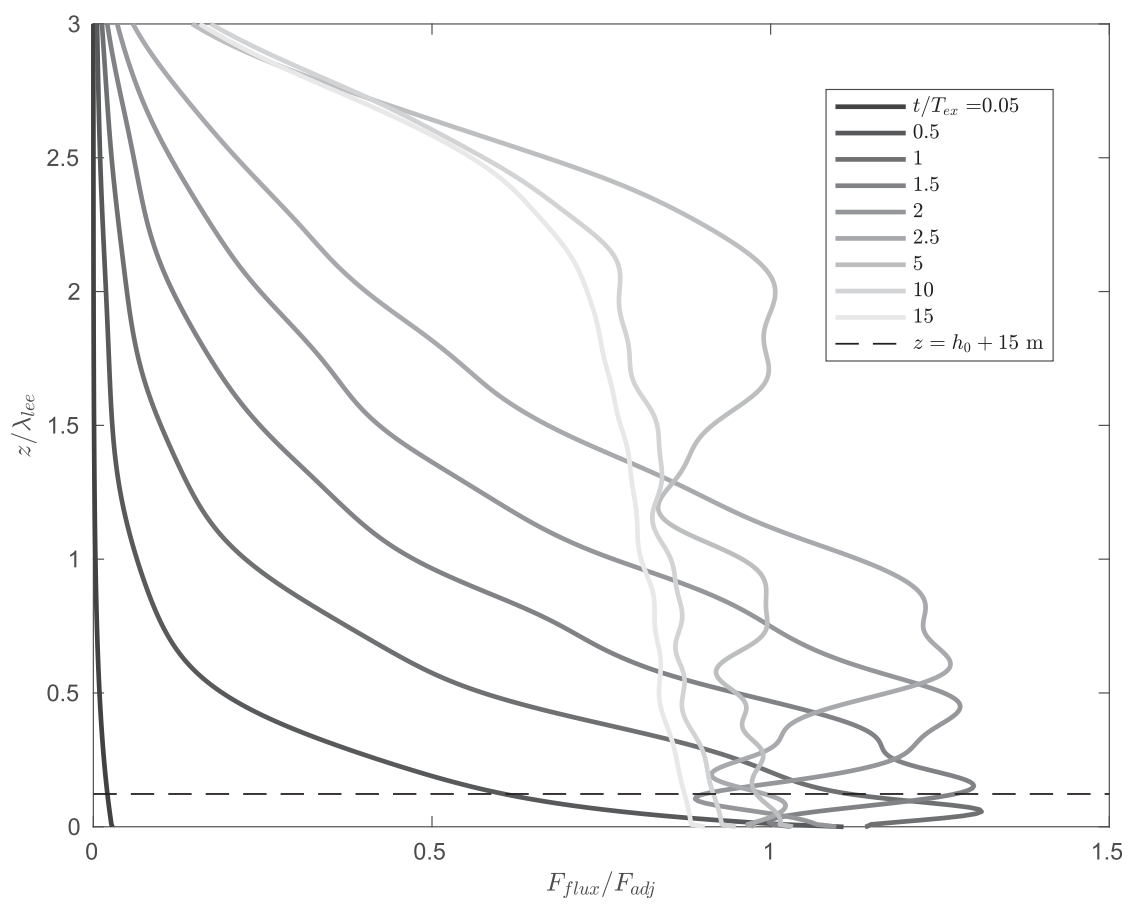

FIG. 5. Horizontally averaged vertical momentum flux as a function of height above the valley bottom for the simulation in Fig. 2 [Eq. (10)]. Each line corresponds to a moment in the simulation, as indicated in the legend. The horizontal dashed line is at the height $h_{0}+15 \mathrm{~m}$ above the valley bottom. 

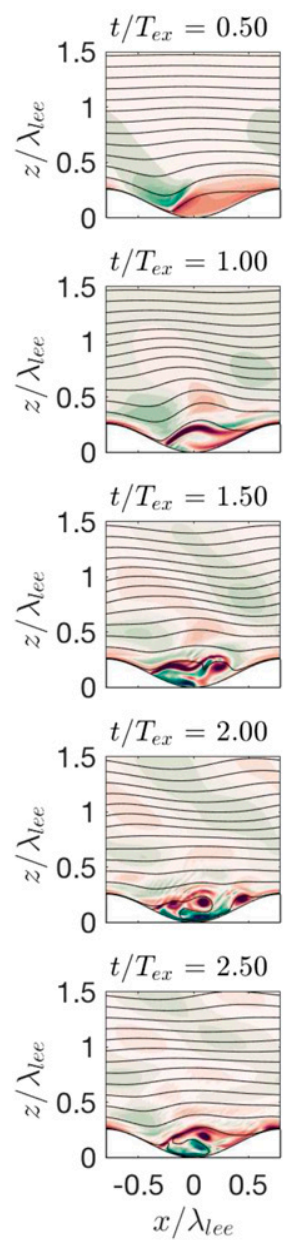

$t / T_{e x}=0.50$

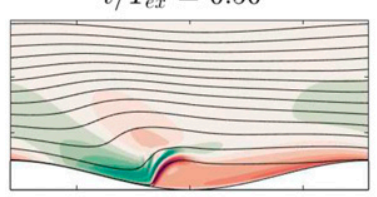

$t / T_{e x}=1.00$

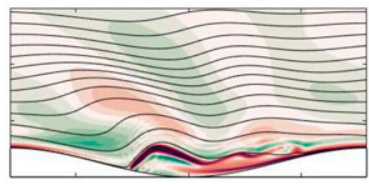

$t / T_{e x}=1.50$

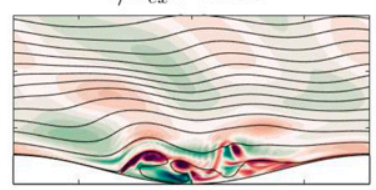

$t / T_{e x}=2.00$

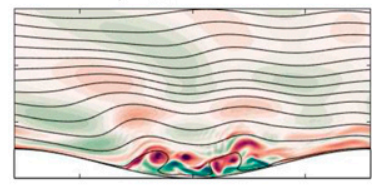

$t / T_{e x}=2.50$

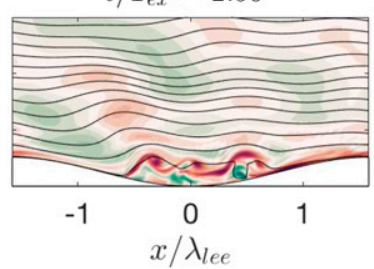

$t / T_{e x}=0.50$

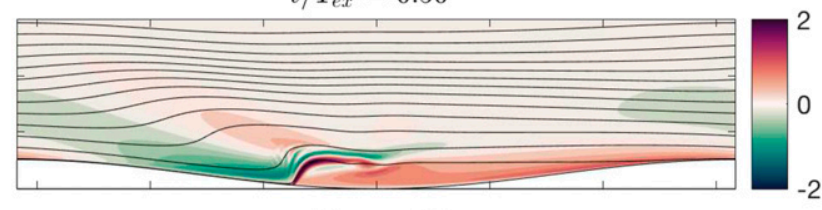

$t / T_{e x}=1.00$

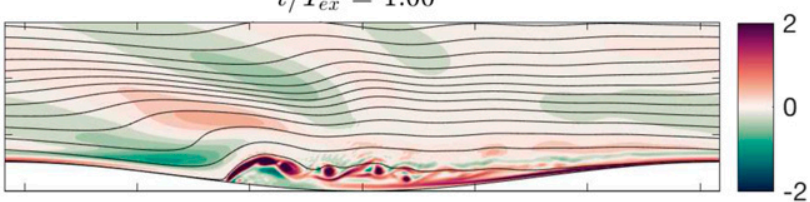

$t / T_{e x}=1.50$

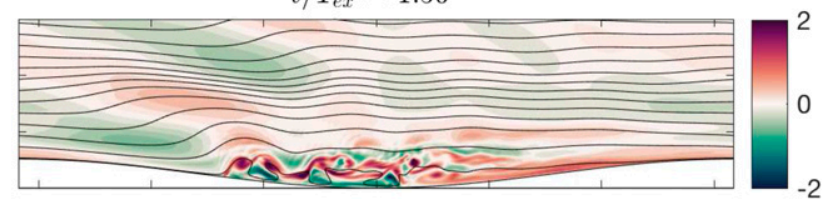

$t / T_{e x}=2.00$

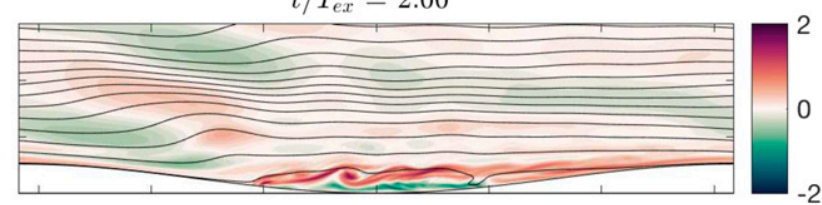

$t / T_{e x}=2.50$

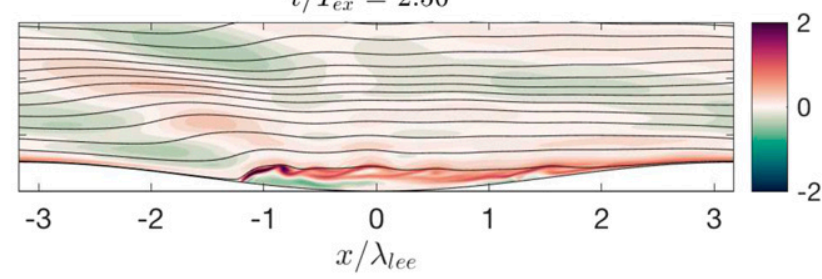

FIG. 6. Snapshots in time of nondimensional vorticity $\left[\omega^{*}=\omega /(J N)\right]$ and streamlines at half $T_{\mathrm{ex}}$ intervals for the simulations with $J=1.6$ and $\epsilon=[0.63,0.32,0.16]\left(h_{0}=160 \mathrm{~m}, L_{\text {hill }}=[1,2,4] \mathrm{km}\right)$. All panels employ real aspect ratios and have their axes nondimensionalized by the lee wave wavelength, $\lambda_{\text {lee }}=2 \pi U / N$.

drag in the vertical rather than always assigning it to the bottom layer.

\section{b. Supercritical cases $(J>1)$}

In this section we report on the temporal evolution of three supercritical simulations with hills of constant height, $h_{0}=160 \mathrm{~m}(J=1.6)$, but different length, $L_{\text {hill }}=$ $[4,2,1] \mathrm{km}(\epsilon=[0.16,0.32,0.63])$. This subset illuminates both similarities across supercritical lee waves with different values of $\epsilon$ and important quantitative discrepancies in the form drag, especially for the narrowest hill $(\epsilon=0.63)$.

The temporal evolution of vorticity and streamlines over the first $2.5 T_{\mathrm{ex}}$ for the three supercritical hills (Fig. 6) demonstrates a remarkable qualitative similarity between lee waves over supercritical hills of dramatically different widths. In all three simulations, the lowest parcels of water become blocked and induce separation on the downslope face within the first half excitation period. Within another excitation period, the shear between the overtopping flow and the blocked layer evolves into a train of vortices that populate the valley. Thereafter, these qualitative features of the blocked layer change little. Thus, the excitation period $T_{\mathrm{ex}}$ again offers a reasonable time scale for the lee wave to reach steady state.

More quantitatively, Fig. 7 shows the form drag, $F_{\text {form }}$ [Eq. (9)], and the vertical momentum flux through a horizontal plane $15 \mathrm{~m}$ above the hill, $F_{\text {flux }}$ [Eq. (10)], for $0 \leq t \leq 6 T_{\text {ex }}$. All three simulations exhibit large discrepancies between the two measures of drag during $t<$ $T_{\text {ex }}$, indicating significant momentum deposition during this period. After the first $T_{\mathrm{ex}}$, however, the simulations display nearly balanced form drags and momentum fluxes, implying that, just as in the subcritical example, the momentum within the control volume is only altered 


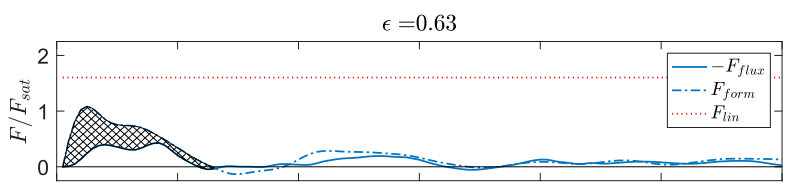

$\epsilon=0.31$
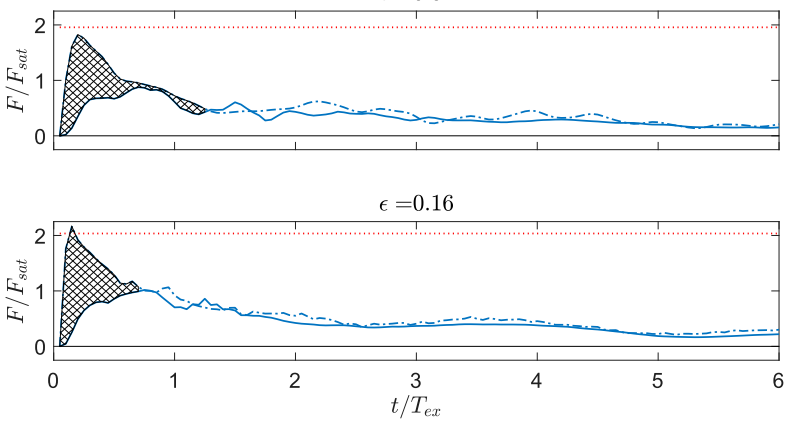

FIG. 7. Comparison of vertical momentum flux through a horizontal plane $15 \mathrm{~m}$ above the crest of the hill, $F_{\text {flux }}$ [Eq. (10)], to the form drag $F_{\text {form }}$ [Eq. (9)]. The values are nondimensionalized by the saturation drag $F_{\text {sat }}=\rho_{0} U^{3} N^{-1}$. Cross hatch pattern indicates local deposition of momentum during spinup [Eq. (12)].

during the first $T_{\text {ex }}$ of the simulation [Eq. (11)]. For the two longer hills $(\epsilon=[0.16,0.31]), F_{\text {form }}$ and $F_{\text {flux }}$ stabilize at around $0.4 F_{\text {sat }}$ [Eq. (5)], in accordance with saturation theory. However, the narrowest hill $(\epsilon=0.63)$ stabilizes at a much smaller magnitude. Above such a narrow hill, the blocked layer quickly fills enough of the valley to render the entire overtopping streamline evanescent, a point to which we return below.

The large discrepancies between the maximum $F_{\text {form }}$ and $F_{\text {flux }}$ during the first $T_{\text {ex }}$ is unique to the supercritical simulations and results in a significant change to the momentum of the fluid within the control volume [Eq. (12)]. Comparison with the flow for $t<2 T_{\mathrm{ex}}$ displayed in the top panels of Fig. 6 encourages the interpretation that the local momentum deposition serves to arrest the valley water and establish the blocked layer, as expected for supercritical periodic bathymetry (Welch et al. 2001). We can estimate the horizontal momentum change $M_{b}^{\prime}$ required to block the valley water as a product of the volume $V_{b}$ contained within the blocked layer and the background velocity $U$, namely,

$$
M_{b}^{\prime}=\rho_{0} V_{b} U .
$$

We compute this value at the time $T_{b}$, which we define as the first instance that $F_{\text {flux }}=F_{\text {form }}$, and compare it to the change in momentum within the control volume during the time period, computed by integrating Eq. (12) from $t=0$ to $t=T_{b}$. For these three hills $(\epsilon=[0.63,0.32,0.16])$, the comparison gives the ratios $M_{b}^{\prime} / M^{\prime}=[1.23,0.92,0.92]$. This close equivalence confirms that the imbalance in the form drag and the vertical momentum flux during the spinup of these supercritical lee waves indeed serves to establish the blocked layer.

The observation that for the two longer hills $(\epsilon=$ $[0.16,0.31])$, the maximum value of the form drag is well approximated by the prediction of linear theory, $F_{\text {lin }}$ [Eq. (3)], while the momentum flux instead peaks around the saturation value $F_{\text {sat }}=\rho_{0} U^{3} N^{-1}$ [Eq. (5)], as predicted by saturation theory, suggests that a scale for the local momentum deposition is given by $M_{b}^{\prime} \sim T_{\text {ex }}\left(F_{\text {lin }}-F_{\text {sat }}\right)$. To test this observation, Fig. 8 displays contour plots of the maximum computed vertical momentum flux and form drag for all simulations in this study, as well as the linear prediction [Eq. (3)]. The close correspondence between the maximum computed form drag and the prediction from linear theory is remarkably robust across most of the $J-\epsilon$ regime spanned by the simulations, especially for $\epsilon \leq$ 0.5 . Likewise, the vertical momentum flux displays ubiquitous saturation of $O\left(F_{\text {sat }}\right)$. Conceptually, this supports the general conclusion that during the first excitation period the form drag reaches the linear value, but the background conditions imposed by $U$ and $N$ restrict the momentum flux to the saturation value $F_{\text {sat }}$. The extra form drag thus acts to decelerate the valley water and establish a blocked layer. However, because the momentum sink is restricted to the first excitation period of lee wave generation, it is dramatically different from the parameterizations for the nonpropagating drag found in Pierrehumbert (1987) and Garner (2005), for which the local momentum sink is assumed to persist into steady state.

Finally, in Fig. 9 we show the evolution of the LOTS for the simulations in Fig. 6 for time $t \leq 16 T_{\text {ex }}$. We note that the separated portion of the LOTS in each simulation displays persistent undulations with length scales of approximately $\lambda_{\text {lee }}$. Furthermore, the LOTS evolves very slowly for $t>6 T_{\mathrm{ex}}$, indicating that the simulations indeed approach a quasi-steady state. Thus, in what follows, quantities with the overbar (e.g., $\bar{u}$ ) are time averaged during $6 T_{\mathrm{ex}} \leq t \leq 12 T_{\mathrm{ex}}$.

The time-variable LOTS in Fig. 9 can be used to explain the dramatic reduction in drag observed in the very nonhydrostatic $\epsilon=0.63$ hill after the establishment of the blocked layer. In all supercritical simulations, once the blocked layer is formed, the LOTS has two different sections, one that is defined by the unblocked portion of the bathymetry, and another that is separated from the bathymetry. For the simulation above the narrow $\epsilon=$ 0.63 hill, the separated section of the LOTS comprises more than half of the total LOTS. As discussed in more detail in the next section, the separated portion of the LOTS is evanescent and does not generate lee waves. 

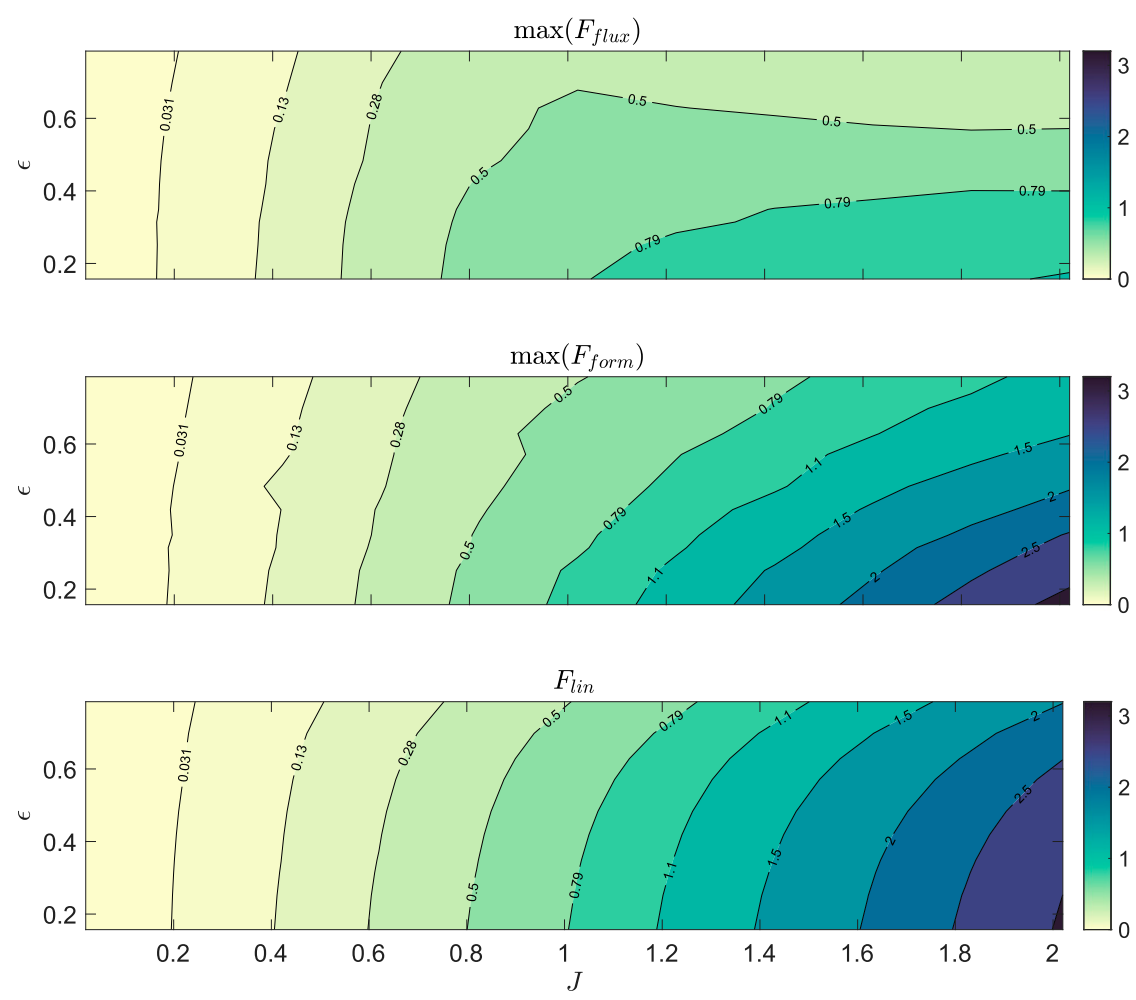

FIG. 8. Maximum computed (top) momentum flux, (middle) form drag, and (bottom) predicted drag from linear theory for all simulations. The values are nondimensionalized by the saturation $\operatorname{drag} F_{\text {sat }}=\rho_{0} U^{3} N^{-1}$.

However, for this narrow hill, the hill length is less than two lee wave wavelengths, as indicated by $\epsilon=\lambda_{\text {lee }} / L_{\text {hill }}>$ 0.5. Therefore the unseparated portion of the LOTS, which comprises less than half of the total LOTS, is also evanescent to the flow, and thus exerts no drag after the establishment of the blocked layer. This process, which we will term "evanescent masking," has dramatic effects on the steady-state drag for all hills in which the unseparated portion of the LOTS is evanescent, as discussed in the next section.

\section{Nonlinear, nonhydrostatic effects on time-averaged drag}

In this section, we analyze the steady-state lee wave drag in our simulations $\bar{F}_{\text {SUN }}$, obtained by averaging $F_{\text {flux }}$ [Eq. (10)] over the period $6 T_{\mathrm{ex}} \leq t \leq 12 T_{\mathrm{ex}}$

$$
\bar{F}_{\text {SUN }}=\frac{1}{6 T_{\text {ex }}} \int_{6 T_{\text {ex }}}^{12 T_{\text {ex }}} F_{\text {flux }} d t
$$

and compare the results with linear and saturation theory. Unless otherwise noted, we use the vertical momentum flux $F_{\text {flux }}$ computed just above the hill [Eq. (10)] rather than the direct computation of the form drag
$F_{\text {form }}$ [Eq. (9)]. The time-averaged drag as a function of $J$ is shown in Fig. 10. In the top panel, the drag is nondimensionalized by $F_{\text {sat }}=\rho_{0} U^{3} N^{-1}$ [Eq. (5)]. This makes apparent that the drag grows with $J$ up to a maximum of roughly $0.5 F_{\text {sat }}$ when $J=1$. We identify blocking as occurring when the maximum vertical excursion of the time-averaged LOTS is less than $95 \%$ of the hill height. With this definition, blocking begins at $J=1$ for the narrowest hills, and is present in all simulations for which $J>1$, confirming the critical Froude number $J_{c}=1$. In the middle panel of Fig. 10, the drags are instead nondimensionalized by the predictions from linear theory $F_{\text {lin }}$ [Eq. (3)]. The effect of blocking in the supercritical simulations is again apparent. However, with this scaling it is evident that the time-averaged drag is smaller than linear theory predicts, even for $J<J_{c}$. The discrepancy in the unblocked cases is a consequence of the reduction of the stratification near the bottom during lee wave spinup, as discussed in section $4 \mathrm{a}$. Indeed, normalizing the drag by $F_{\text {adj }}$ [Eq. (15)] instead of $F_{\text {lin }}$, as in the bottom panel of Fig. 10, removes this trend. It also introduces a new source of noise for the most nonhydrostatic hills, possibly due to the effect of the reduced buoyancy frequency on the vertical wavenumber, which we neglect in computing $F_{\text {adj }}$. Together, 

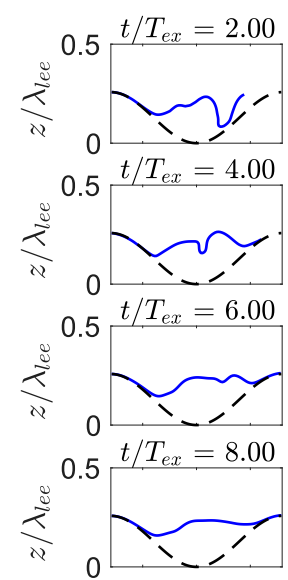

$t / T_{e x}=10.00$
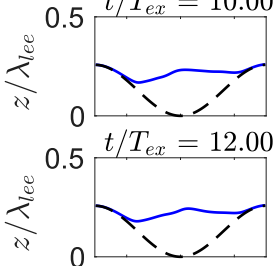

$0.5 t / T_{e x}=14.00$
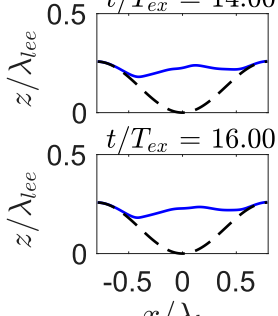

$x / \lambda_{\text {lee }}$
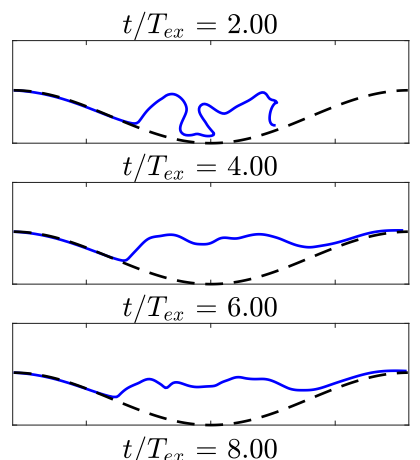

$t / T_{e x}=8.00$
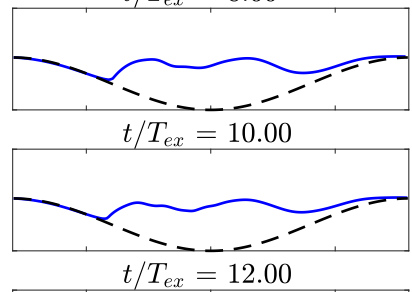

$t / T_{e x}=12.00$

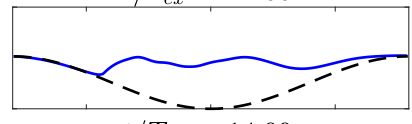

$t / T_{e x}=14.00$

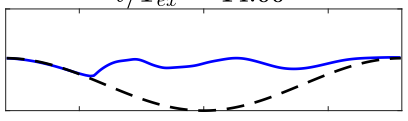

$t / T_{e x}=16.00$

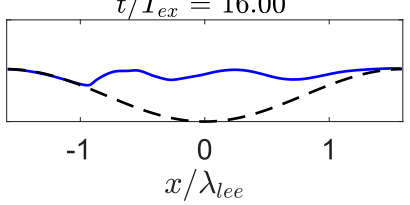

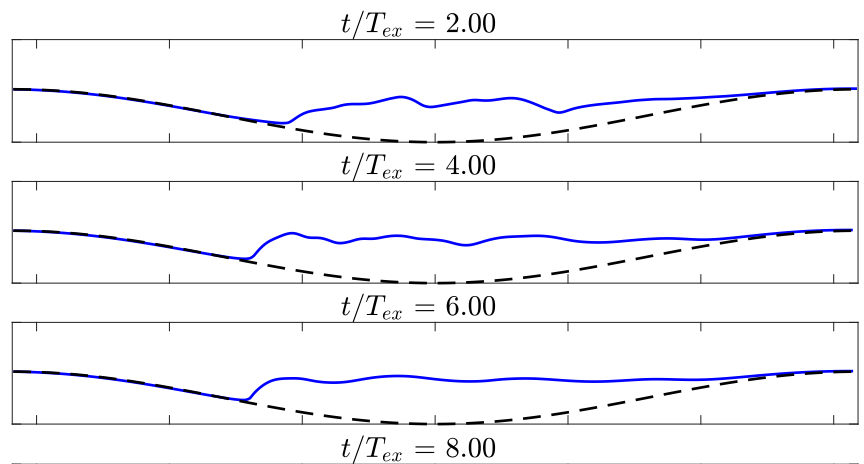
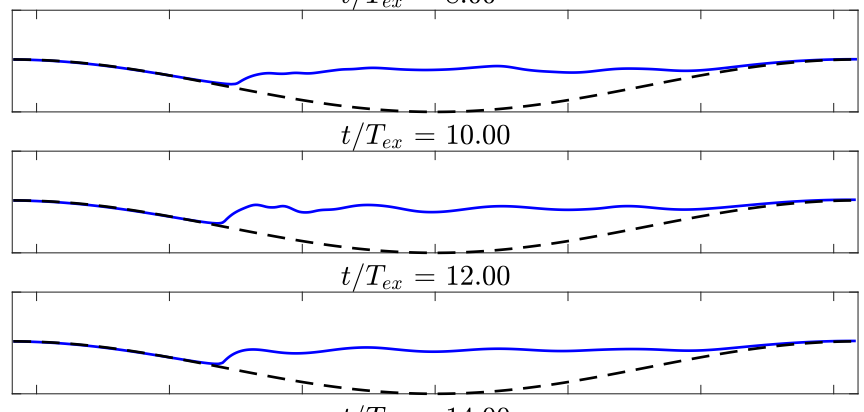

$t / T_{e x}=14.00$

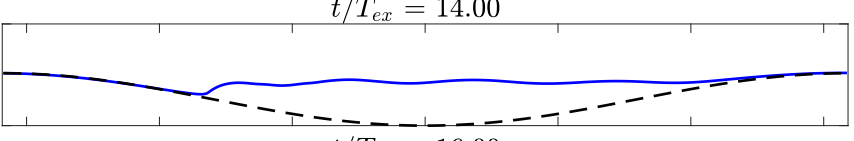

$t / T_{e x}=16.00$

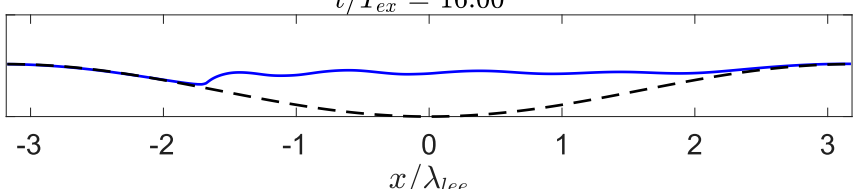

FIG. 9. Snapshots in time of LOTS (solid blue lines) at half $T_{\mathrm{ex}}$ intervals for the simulation with $J=1.6$ and $\epsilon=[0.63,0.32,0.16]\left(h_{0}=\right.$ $\left.160 \mathrm{~m}, L_{\text {hill }}=[1,2,4] \mathrm{km}\right)$. Real bathymetry is shown as dashed black lines.

the three panels demonstrate the separation of the lee wave into three regimes based on hill height: the linear limit $J \ll 1$, where $F_{\text {lin }}$ [Eq. (3)] is accurate, the subcritical regime $J=O(0.1)$, where weak nonlinearities during spinup result in a decay of buoyancy near the bottom and the drag is better predicted by the buoyancy adjusted linear theory $F_{\text {adj }}$ [Eq. (15)], and the supercritical regime $J \geq J_{c}$, in which the lowest parcels of water are trapped in the valleys and the drag is limited by the radiative capacity of the fluid, $F_{\text {sat }}=\rho_{0} U^{3} N^{-1}$ [Eq. (5)].

To demonstrate the dependence of drag on the hill length, Fig. 11 displays the time-averaged drag as a function of $\epsilon$. In general, there is a monotonic decrease in the drag with increasing $\epsilon$, as predicted by linear theory [Eq. (3)]. At moderate values of $\epsilon=[0.57,0.48$, $0.42,0.35]$, however, the critical $J=1$ and supercritical $J=1.2$ height hills demonstrate interesting nonmonotonic behavior (highlighted by squares in Fig. 11). When $J=1$, there is a maximum drag at $\epsilon=0.48$, in contradiction to linear theory. This nonmonotonic behavior can be explained as a consequence of harmonic resonance between the excitation frequency $U k$ and the responding buoyancy frequency $N$. When $\epsilon=0.5, N=2 U k$, implying that the buoyancy frequency is a harmonic of the excitation frequency, amplifying the nonlinear response. On the other hand, when $\epsilon=1 / 3$ or $2 / 3, N=3 U k$ and $3 / 2 U k$, implying a dissonant buoyancy frequency and muted response.

In Fig. 12 we show the correlation between the average computed drag and the drag predicted by buoyancy adjusted linear theory $F_{\text {adj }}$ [Eq. (15)]. We use $F_{\text {adj }}$ rather than $F_{\text {lin }}$ to remove the effect of the reduced buoyancy frequency near the bottom on the predicted drag. From Fig. 12, it is clear that using linear theory based on the length and height scales of the bathymetry is valid in the linear and weakly nonlinear regimes, but fails as predicted by saturation theory when $J=O(1)$. Figure 12 also suggests that the more nonhydrostatic runs (smaller circles) are more strongly affected by saturation. 

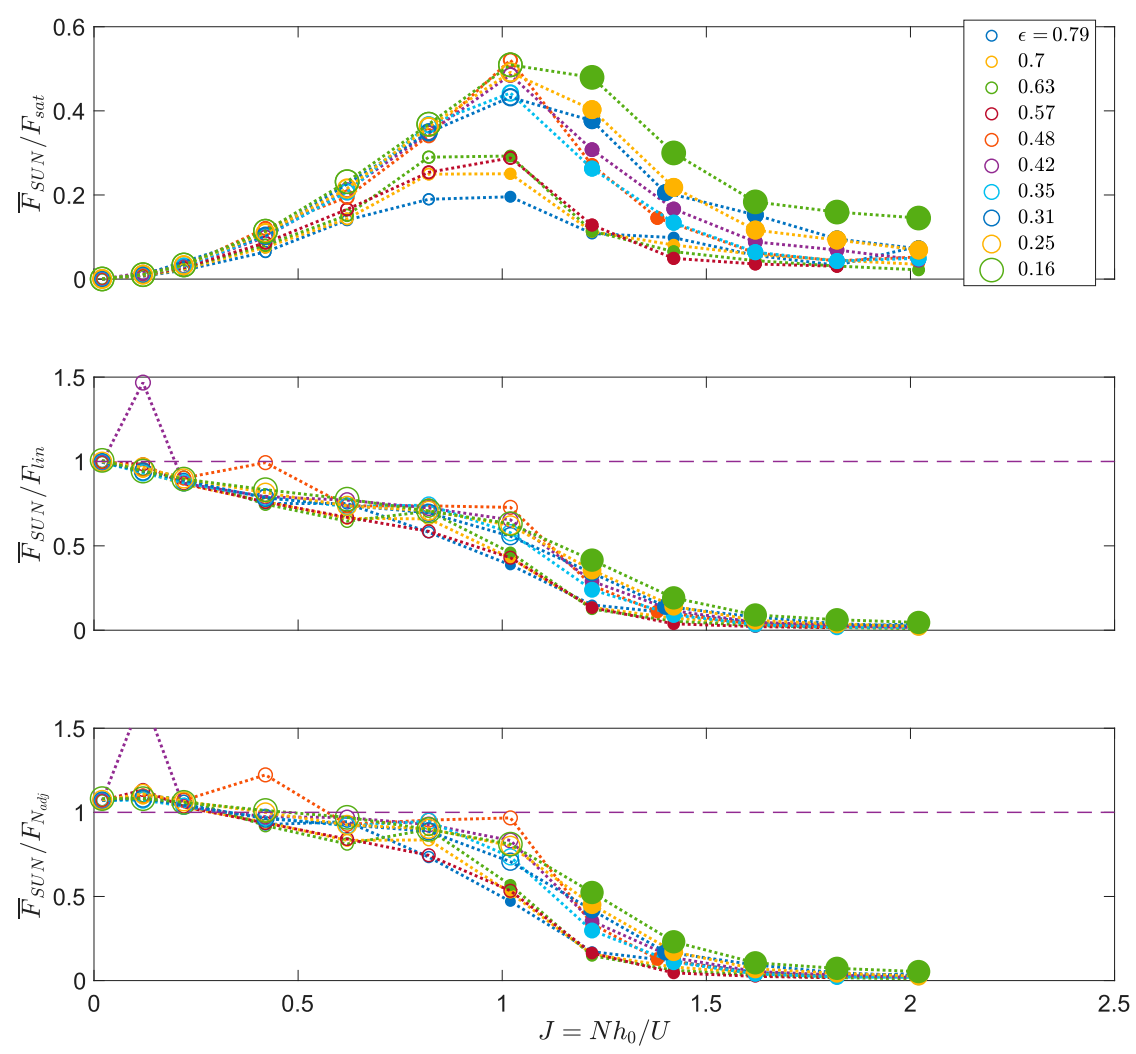

FIG. 10. Time-averaged vertical momentum flux $\bar{F}_{\text {SUN }}$ [Eq. (17)] normalized by (top) $F_{\text {sat }}=$ $\rho_{0} U^{3} N^{-1}$ [Eq. (5)], (middle) $F_{\text {lin }}$ [Eq. (3)], and (bottom) $F_{\text {adj }}$ [Eq. (15)] as a function of $J$ for all simulations. Each simulation is represented by a circle. The size of the circle indicates the relative length of the hill, while filled circles indicate blocking.

As a measure of the strength of the correlation between simulations and parameterizations or theory, we use the coefficient of determination, defined as

$$
R^{2}=1-\frac{\mathrm{SS}_{\mathrm{res}}}{\mathrm{SS}_{\mathrm{tot}}}
$$

where

$$
\mathrm{SS}_{\mathrm{tot}}=\sum\left(y_{i}-\bar{y}\right)^{2}
$$

is the sum of squared errors between the simulated values $y_{i}$ and their mean $\bar{y}$, and

$$
\mathrm{SS}_{\mathrm{res}}=\sum\left(y_{i}-f_{i}\right)^{2}
$$

is the sum of squared differences between the simulated values $y_{i}$ and the parameterized values $f_{i}$. A parameterization that perfectly predicts the simulated value will give $R^{2}=1$, while a negative value of $R^{2}$ indicates that the observed values are better predicted by their mean than by the parameterization. The coefficients of determination for all steady-state parameterizations considered in this section are shown in Table 1. For linear theory using the adjusted buoyancy frequency (Fig. 12), the coefficient of determination is $R^{2}=-53.1$. The poor performance results from using the bathymetric height to scale the drag in supercritical lee waves.

Figure 13 shows the time-averaged LOTS for the simulations in Fig. 9. As posited by saturation theory, the LOTS is the effective bathymetry that generates lee waves, which can be used to compute the effective bathymetric power spectrum and thus predict the drag with the Fourier synthesis of $F_{\text {lin }}$ [Eq. (3)], as in Bell (1975b). Because this method employs all of the horizontal information in the LOTS, this operation represents a fully nonhydrostatic form of saturation theory. We perform this analysis on each run that exhibits blocking and plot the correlation between the average computed drag $\bar{F}_{\text {SUN }}$ [Eq. (17)] and the drag predicted by $F_{\text {Bell }}$ in the upper-left panel of Fig. 15 . With $R^{2}=0.91$, this combination of spectral linear theory with the LOTS is significantly better than the linear theory that uses the bathymetric height and length $F_{\text {adj }}$, for which $R^{2}=-53.1$ (Fig. 12). This demonstrates the strength of viewing the average LOTS as the effective wave-generating bathymetry. 

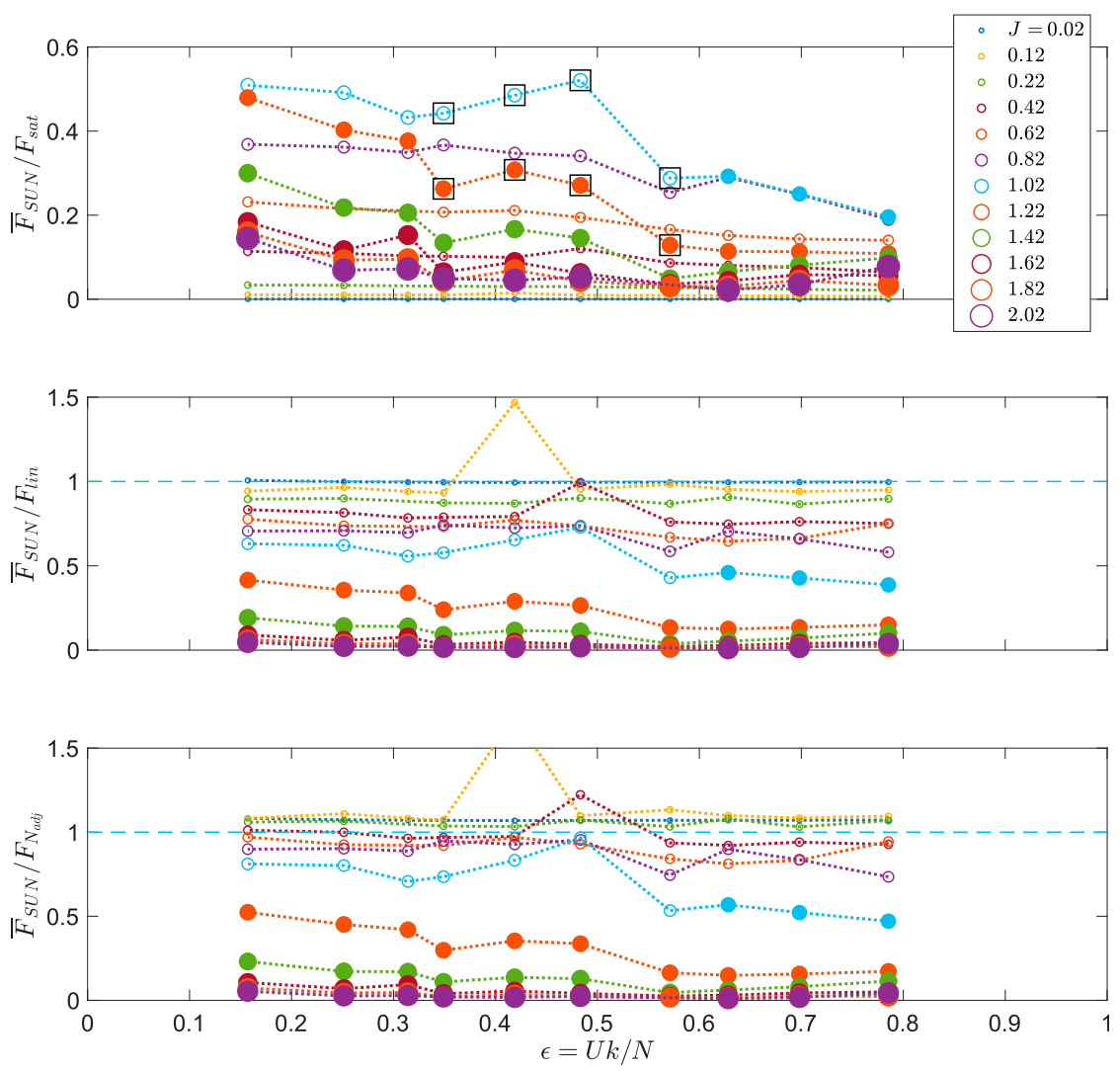

FIG. 11. As in Fig. 10, but as a function of $\epsilon$ for all simulations. The simulations highlighted by squares in the top panel display nonmonotonic behavior due to resonance.

In practice, the power spectrum of the LOTS above abyssal hills is not known a priori. Indeed, the usefulness of hydrostatic saturation theory is its prediction that, for supercritical bathymetry, there exists some average effective hill height of $O(U / N)$ such that the effective wave generating Froude number is $J_{\text {eff }}=O(1)$. To this end, we compute the average effective heights of each LOTS $h_{\text {eff }}$ as indicated in Fig. 14, and define an effective Froude number, $J_{\text {eff }}=N h_{\text {eff }} / U$, to form a simple prediction of the drag based on hydrostatic saturation theory

$$
F_{\text {eff }}=\left(\frac{J_{\text {eff }}}{J}\right)^{2} F_{\text {adj }} .
$$

We perform this analysis on each run that exhibits blocking and compare the computed drag to the drag predicted by $F_{\text {eff }}$ in the upper-right panel of Fig. 15 . Although using hydrostatic saturation theory to compute $F_{\text {eff }}$ gives a better prediction than linear theory (which has $R^{2}=-53.1$; see Table 1 ), with $R^{2}=-0.296, F_{\text {eff }}$ is still less predictive than the mean of the observations. It also uniformly over predicts the lee wave drag.

The discrepancy between the performance of spectral saturation theory $F_{\text {Bell }}$ and hydrostatic saturation theory
$F_{\text {eff }}$ suggest that there is a characteristic component of the supercritical LOTS that the latter neglects. We hypothesize that one important feature of the LOTS is the undulations downstream of the separation points with

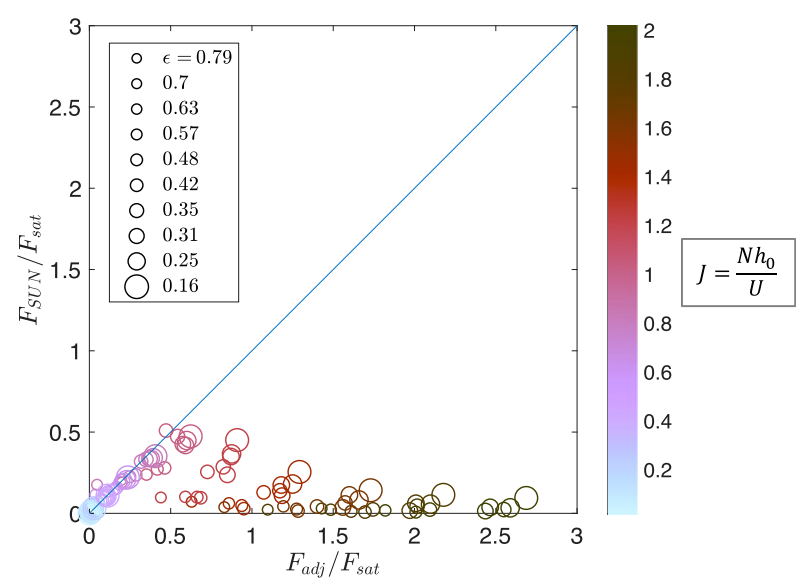

FIG. 12. Comparison of time-averaged vertical momentum flux $\bar{F}_{\text {SUN }}[$ [Eq. (17)] to the prediction from buoyancy-adjusted linear theory $F_{\text {adj }}$ [Eq. (15)]. For each simulation, $J$ and $\epsilon$ are indicated by, respectively, the color and the size of the data point. The coefficient of determination is $R^{2}=-53.1$. 
TABLE 1. Table of coefficient of determination $R^{2}$ values for all steady-state parameterizations considered in section 5 using $N_{\text {adj }}$ [Eq. (14)], as well as the values obtained by using $N$ instead. Since $F_{\text {aloft }}$ only uses $N$, the two values for $F_{\text {aloft }}$ are identical.

\begin{tabular}{lcc}
\hline \hline & $N_{\text {adj }}$ & $N$ \\
\hline$F_{\text {lin }}$ & -53.1 & -83.4 \\
$F_{\text {Bell }}$ & 0.91 & 0.305 \\
$F_{\text {eff }}$ & -0.296 & -1.55 \\
$F_{\text {prop }}$ & 0.733 & 0.146 \\
$F_{\text {aloft }}$ & 0.956 & 0.956 \\
\hline
\end{tabular}

wavelengths roughly equal to $\lambda_{\text {lee }}$. If these undulations are treated as independent periodic bathymetry, they represent hills with $\epsilon \approx 1$ and are thus evanescent and should not contribute to the drag. The total effective height of the LOTS can therefore be partitioned into a propagating $h_{\text {prop }}$ and a nonpropagating $h_{\text {nonprop com- }}$ ponent, as illustrated in Fig. 14. Note that we define $h_{\text {nonprop }}$ as the maximum height of the undulations above the blocked layer rather than as the height of the jump-like feature at the beginning of the blocked layer because only the former of these features can be treated as independent periodic bathymetry. In this sense, the separation of $h_{\text {eff }}$ into $h_{\text {prop }}$ and $h_{\text {nonprop }}$ represents an approximation of the power spectrum of the LOTS by two primary components. Only the propagating component contributes to the drag, suggesting that

$$
F_{\text {prop }}=\left(\frac{J \text { prop }}{J}\right)^{2} F_{\text {adj }},
$$

where

$$
J_{\text {prop }}=\frac{N}{U}\left(h_{\text {eff }}-h_{\text {nonprop }}\right) .
$$

The bottom-left panel of Fig. 15 compares the computed drag to $F_{\text {prop. }}$. It performs much better than hydrostatic saturation theory with $R^{2}=0.733$, indicating that the evanescent undulations are indeed dynamically relevant. However, $F_{\text {prop }}$ is not as skilled as spectral saturation theory, and still tends to overestimate the drag.

The signal of an evanescent lee wave decays with height above the ocean floor at a rate determined by the vertical wavenumber [Eq. (1)]. Hence, a streamline sufficiently far from the bathymetry should contain contributions from only the propagating component of the lee wave. This observation leads to a final parameterization for the effective bathymetry, with an effective height $h_{\text {aloft }}$ based on the maximum vertical displacement of a streamline originating at $z=h_{0}+\lambda_{\text {lee }} / 2$ above the crest of the hill (red dotted lines in Fig. 13). The
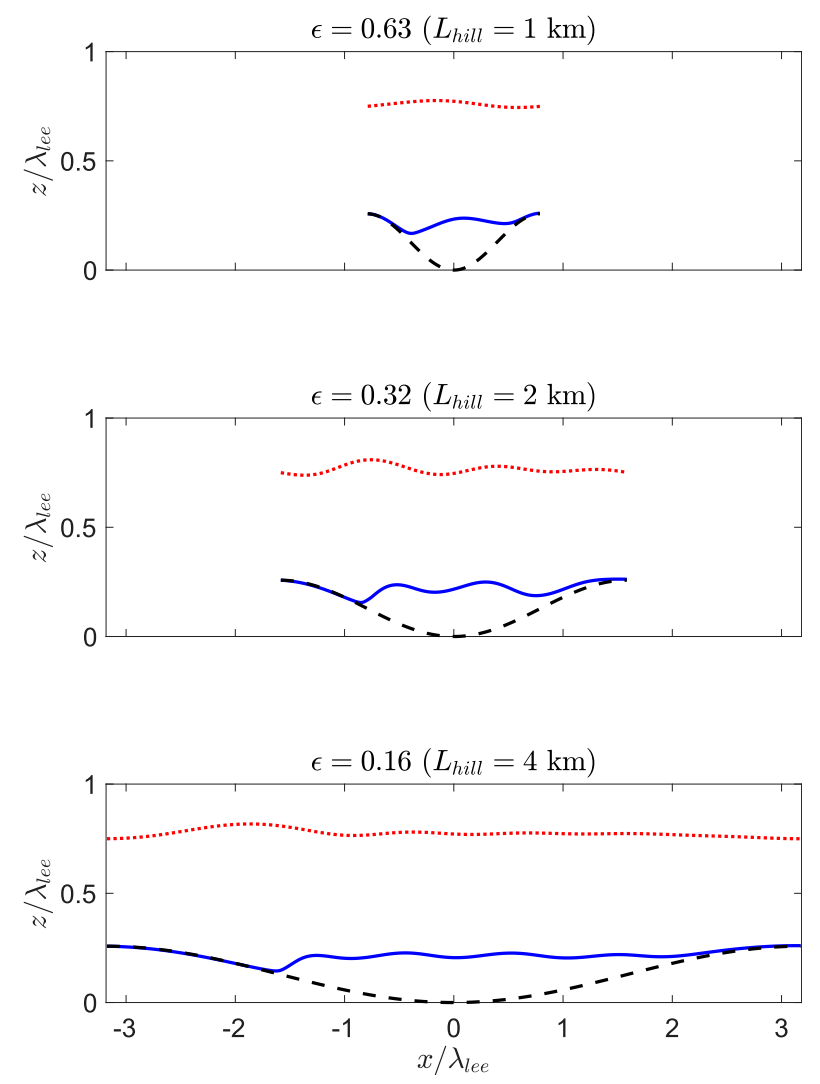

FIG. 13. Time-averaged LOTS (blue solid line) and streamlines starting at $z=h_{0}+0.5 \lambda_{\text {lee }}$ (red dotted lines) corresponding to the simulations shown in Fig. 6. The aspect ratio is 2:1 to accentuate vertical perturbations.

height $h_{\text {aloft }}$ implies a new Froude number, $J_{\text {aloft }}=$ $N h_{\text {aloft }} / U$, and a new parameterization for the drag

$$
F_{\text {aloft }}=\left(\frac{J_{\text {aloft }}}{J}\right)^{2} F_{\text {lin }} .
$$

As shown in Fig. 15, this drag prediction agrees remarkably well with the computed drag, with a coefficient of determination of $R^{2}=0.956$. Similarly excellent performance (not shown) results from selecting a streamline twice as far from the bottom. This parameterization has the added advantage that it is insensitive to the decay of buoyancy near the bottom, since the streamline upon which $J_{\text {aloft }}$ is based is at a height above the observed decay. Furthermore, parameterizing the drag with the aloft streamline does not require the spectrum of the streamline, as in the calculation of $F_{\mathrm{Bell}}$, only its maximum vertical excursion $h_{\text {aloft. The combination of simplicity and }}$ accuracy in this parameterization suggests that a good estimate of the lee wave drag in the ocean may be obtained simply by measuring the vertical excursion of a streamline at a height $z_{\text {aloft }}=\pi U / N$ above the bathymetry. 


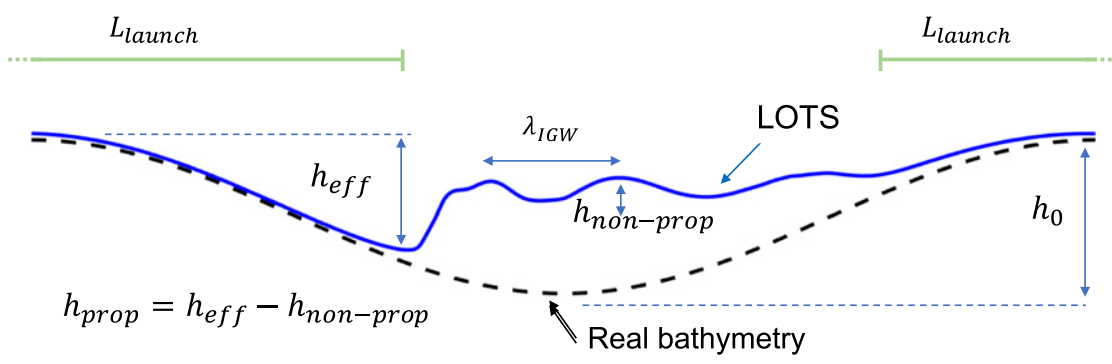

FIG. 14. Sketch of the effective bathymetry (LOTS) and its characteristic height and length scales from the simulation with $J=1.6$ and $\epsilon=0.16$.

Upon averaging across all of the simulations that display blocking (indicated by a tilde), we observe an average effective Froude number of $\tilde{J}_{\text {eff }}=0.7 \pm 0.07$, an average propagating Froude number of $\tilde{J}_{\text {prop }}=0.5 \pm 0.06$, and an average aloft Froude number of $\tilde{J}_{\text {aloft }}=0.4 \pm 0.09$. Unlike most versions of saturation theory, in which $J_{\text {eff }}=J_{c}$, here all values for the effective Froude number are smaller than the observed critical Froude number $J_{c}=1$, indicating that a supercritical lee wave has a smaller drag than a critical lee wave. Our values of $\tilde{J}_{\text {eff }}$, $\tilde{J}_{\text {prop }}$, and $\tilde{J}_{\text {aloft }}$ are also significantly smaller than the effective Froude number of Nikurashin and Ferrari (2010), $J_{\mathrm{NF}}=1.4$ (this accounts for their definition of hill height, which is half of ours, i.e., $h_{0}=2 h_{\mathrm{NF2} 2010}$ ).
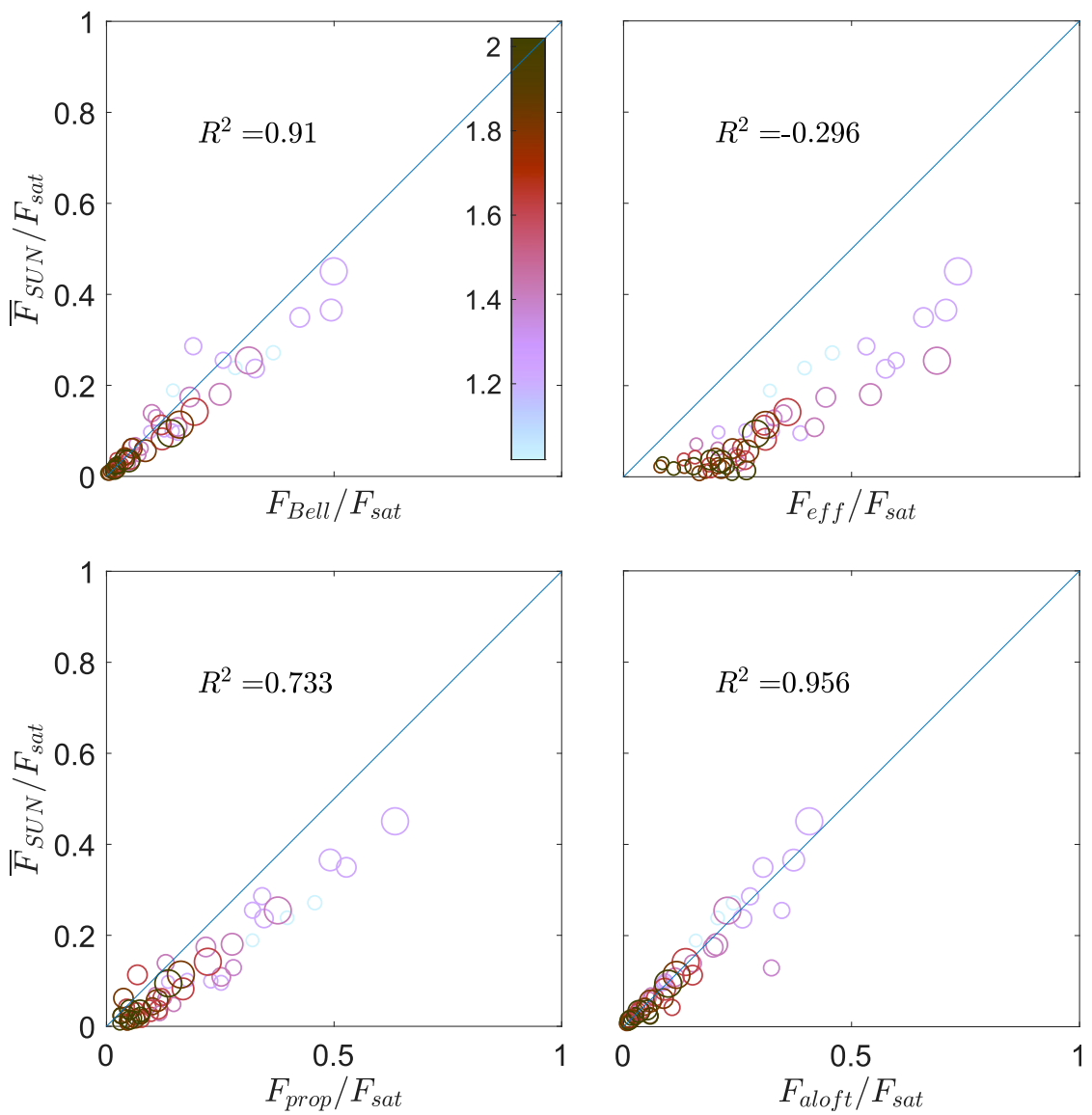

FIG. 15. As in Fig. 12, but now comparing to (top left) $F_{\text {Bell }}$, (top right) $F_{\text {eff }}$ [Eq. (21)], (bottom left) $F_{\text {prop }}$ [Eq. (22)], and (bottom right) $F_{\text {aloft }}$ [Eq. (24)]. Only the simulations that exhibit blocking are displayed. The coefficients of determination [Eq. (18)] are indicated in each panel. 

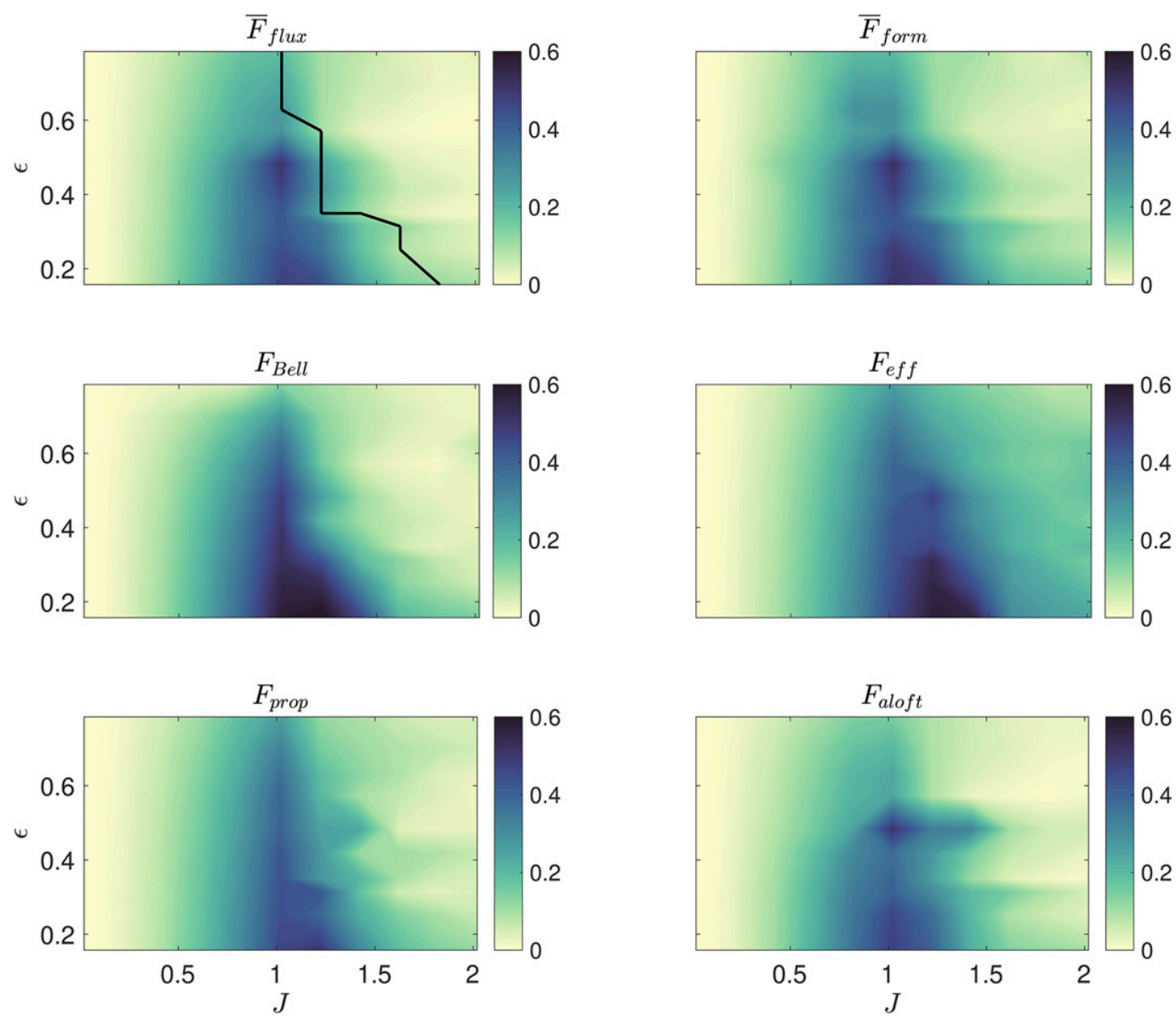

FIG. 16. Parameterized and time-averaged drags for all simulations. The black line plotted with $\bar{F}_{\text {flux }}$ (in the top-left panel) shows the drag-free boundary predicted by evanescent masking [Eq. (25)].

Thus, the nonhydrostatic corrections to simple saturation theory embodied by $F_{\text {prop }}$ and $F_{\text {aloft }}$ suggest that the lee wave drag over supercritical abyssal hills could be $50 \%$ smaller than currently thought. Furthermore, since $J_{\text {aloft }}<J_{c} / 2$, these simulations suggest the most intense abyssal lee waves occur instead over critical and nearly critical abyssal hills, before the onset of blocking.

To summarize this section, Fig. 16 shows contour plots in $J-\epsilon$ space of the two measures of time-averaged drag $\left(\bar{F}_{\text {flux }}\right.$ and $\left.\bar{F}_{\text {form }}\right)$ and the four supercritical parameterizations for drag discussed in this section $\left(F_{\text {eff }}, F_{\text {Bell }}\right.$, $F_{\text {prop }}$, and $\left.F_{\text {aloft }}\right)$. Comparison of $\bar{F}_{\text {flux }}$ and $\bar{F}_{\text {form }}$ show them to be identical $\left(R^{2}=0.97\right)$, indicating that just as in the linear regime of Pedlosky (2003), during the majority of the supercritical lee wave event over periodic bathymetry, the form drag and the vertical momentum flux are in balance, and there is no change in momentum near the ocean floor.

Figure 16 also highlights a curious $\epsilon$-dependent feature that is not predicted by hydrostatic saturation theory, namely, the precipitous decline in simulated drag when $J>1$ and $\epsilon>0.5$. We hypothesize that this drop off is due to evanescent masking, wherein the blocked layer grows horizontally to the point in which the attached portion of the LOTS becomes evanescent in length. Defining the horizontal length of this unseparated portion as $L_{\text {launch }}$ (see Fig. 14), we thus expect evanescent masking when

$$
L_{\text {launch }}<\lambda_{\text {lee }} \text {. }
$$

The boundary predicted upon measuring $L_{\text {launch }}$ in every simulation is sketched in the top-left panel of Fig. 16, and aligns remarkable well with the observed drop-off in drag. Evanescent masking thus represents a second nonhydrostatic process present in oceanic lee waves above periodic bathymetry which is also absent from current implementations of saturation theory. Like the evanescent undulations over the blocked layer, evanescent masking has the effect of reducing the lee wave drag above supercritical hills below that predicted by 
saturation theory. Indeed, for the most supercritical simulations in this study, evanescent masking renders even the hydrostatic hills practically evanescent.

\section{Conclusions}

In this paper, we used idealized simulations of lee waves over one-dimensional sinusoidal bathymetry to demonstrate that for oceanic flow over supercritical height bathymetry, the lowest layer of fluid becomes blocked and presents the overtopping flow with an effective bathymetry defined by the lowest overtopping streamline (LOTS) that is subcritical in height and polychromatic in wavelength. That the effective bathymetry reduces the apparent height of the hills is well known and currently employed in parameterizations of lee wave drag in global ocean models. However, both the development of evanescent undulations in the LOTS above the blocked layer and the process of evanescent masking, in which the unseparated portion of the LOTS becomes narrower than the lee wave wavelength, are novel results of nonhydrostatic lee wave processes in this paper. Recognizing their deleterious effect on the wave drag in regions of supercritical $(J \geq 1)$ bathymetry may help explain the discrepancy between predictions using existing parameterizations and observation of lee wave activity in the ocean discussed in Kunze and Lien (2019).

This study also offered an opportunity to observe the spinup of weakly nonlinear $[J=O(0.1)]$ and supercritical $(J \geq 1)$ lee waves above periodic bathymetry. The excitation frequency, $T_{\mathrm{ex}}=L_{\mathrm{hill}} / U$, emerged as the fundamental time scale of the lee wave for all simulations, regardless of hill height or length, informing both the time required for spinup and the vertical location of the drag on the background current. Furthermore, analysis of the horizontal momentum budget during spinup of supercritical lee waves permitted a characterization of timedependent local drag associated with blocking.

The results in this paper identify four notable deficiencies in existing parameterizations of the lee wave drag. 1) In supercritical regions $\left(J \geq J_{c}\right)$, the simulations suggest that the steady-state lee wave drag will be smaller than predictions with existing saturation parameterizations due to the omission of nonhydrostatic effects of evanescent undulations and evanescent masking. 2) We observe that the drag associated with blocking is constrained to the first excitation period when the blocked layer forms, whereas predictions of the "nonpropagating" drag given by Garner (2005) and used in Trossman et al. $(2013,2015,2016)$ assume the blocking component of the drag is a steady process that continually removes momentum from the flow. 3) On the other hand, in regions with large but still subcritical height bathymetry $\left(J<J_{c}\right)$, our parameterizations predict larger lee wave drag because we identify the critical Froude number $J_{c}$ and the effective Froude number $J_{\text {aloft }}$ separately, whereas existing saturation theory assumes them to be identical. 4) Finally, our observations of upward-propagating lee wave wave fronts in every simulation suggest that all existing parameterizations need to reconsider confining the drag to within one lee wave wavelength above the bathymetry.

There are inevitably some limitations in applying our conclusions uniformly throughout the ocean. First, there is inherent uncertainty of approximately $30 \%$ in both the local height and length of abyssal hills in the Goff and Arbic (2010) abyssal hill bathymetry product. On a regional scale, this uncertainty is mitigated by the statistical homogeneity of the abyssal hills. Nevertheless, such significant local uncertainty of abyssal hill length and width suggests that some of the details in our process study, such as that of resonant responses for the $J=1$ simulations, are probably not worth including in a drag parameterization.

Second, the predicted drags $F_{\text {adj }}, F_{\text {Bell }}, F_{\text {eff }}, F_{\text {prop }}$, and $F_{\text {aloft }}$ presented in section 5 required running the simulations to find the effective Froude numbers and the adjusted buoyancy frequencies $N_{\text {adj }}$, meaning that these were not a priori parameterizations of the drag. With respect to the effective Froude numbers, the aloft Froude number $J_{\text {aloft }}$ gave the most accurate prediction of the lee wave drag. Upon averaging across all supercritical simulations, we found the average aloft Froude number of $\tilde{J}_{\text {aloft }}=0.4 \pm 0.09$. The variability in this average stems primarily from resonant responses rather than from nonhydrostatic effects. Hence our results support lee wave drag parameterizations replacing $J_{\mathrm{NF}}=1.4$ with $J_{\text {aloft }}=0.4$ above all two-dimensional abyssal hill bathymetry. Such a parameterization could also approximate the nonhydrostatic effect of evanescent masking [Eq. (25)] with a low-pass filter on the abyssal hill power spectrum to include only hills for which $\epsilon<0.5$. Using our results to parameterize $N_{\text {adj }}$ is more difficult. As discussed in section 4, the decay of near-bottom buoyancy appears to depend on the progression of the valley water over the top of the hill during the first excitation period of the simulation. This suggests that $N_{\text {adj }}$ is, at a minimum, a function of $N, U, h_{0}$, and $k$. Additionally, it involves mixing, which for our simulations is a process driven by the elevated kinematic viscosity. In the real ocean, there are likely further complications such as effects of the bottom boundary layer and hysteresis from previous lee wave events. Hence the parameterization of $N_{\text {adj }}$ requires further research.

Third, our study focused on single wavelength bathymetry, while the abyssal hills are better described 
by a linear superposition of many wavelengths. As such, our study cannot account for nonlinear interactions imposed by polychromatic nonlinear bathymetry. One hypothesis is that the flow will adjust by filling in all valleys with stretches of evanescent undulations as observed above, whereafter the remaining unblocked peaks will likely represent a subset of the original bathymetric wavenumbers. However, the hypothesis has not yet been tested with simulations. This is a significant limitation when attempting to employ the Goff and Arbic (2010) abyssal hill product.

Fourth, our study is of two-dimensional flow over onedimensional bathymetry. In three-dimensional flow with two-dimensional bathymetry, fluid can travel around an obstacle rather than over it, and has been shown to reduce the saturation momentum flux by $40 \%$ (Nikurashin et al. 2014). However, abyssal hills are generally anisotropic, presenting the flow with a corrugation of ridges rather than a field of seamounts, which suggests that there may be little opportunity for flow around rather than over them.

Finally, our study ignores rotation. A primary consequence of rotation on lee waves is the lower bound on the bathymetric wavenumbers that produce propagating lee waves, based on the criterion $f<U k$. As $U k \rightarrow f$, the vertical wavenumber approaches infinity, whereupon the vertical group velocity and momentum flux vanish. Hence, our conclusions about the drag for an upward propagating lee wave require modification for longer wavelength hills in strongly rotational regions. A second rotational process that complicates our conclusions is that of lee wave frequency-band narrowing, resulting from the near-bottom decay of $N$ during spinup of nonlinear lee waves [Eq. (14)] such that the window of drag-producing bathymetry shrinks according to $f<$ $U k<N_{\text {adj }}<N$. If dramatic enough, or in regions of strong rotation, it could make all lee waves evanescent, snuffing out the lee wave drag (Kunze and Lien 2019). Finally, Nikurashin and Ferrari (2010) demonstrate that in the Southern Ocean where rotation is strong, saturation-level velocities in the lee wave field are sufficient to excite inertial oscillations and elicit positive feedback that results in wave breaking within the first lee wave wavelength above the bathymetry and a local deposition of momentum. Thus, in strongly rotational regions it may not be appropriate to deposit the momentum according to the vertical group velocity. However, even in this regime, the low-level breaking is a slowly developing process. Nikurashin and Ferrari (2010) observe the need for five or more days for inertial oscillations to grow over subcritical bathymetry even though the excitation period for their bathymetry was approximately just five hours. Therefore, there is ample time for the lee wave wave front to propagate away from the bottom, depositing momentum as it travels before the development of instabilities related to the inertial oscillations.

Despite the many simplifications, this paper demonstrates the remarkable applicability of linear lee wave theory to real ocean-scale flow. During spinup, $F_{\text {lin }}$ [Eq. (3)] offered a good estimate for the peak value of the form drag in all simulations, and was especially accurate when $\epsilon<0.5$, where evanescent masking is less pronounced. After spinup, the observed quasi-steady wave drag was best predicted with the same linear theory but with the hill height replaced with the maximum vertical extent of a streamline aloft $F_{\text {aloft }}$ [Eq. (24)]. The second best prediction came from inserting the power spectrum of the effective bathymetry into the spectral form of linear lee wave drag (Bell 1975b). These should not be foregone conclusions, since linear theory is strictly valid only for $J \ll 1$, whereas the effective bathymetries in our simulations presented effective lee wave Froude numbers of $J_{\text {aloft }} \approx 0.4$. Granted, achieving such accurate predictions required simulating the full flow to compute the adjusted bottom buoyancy frequency and the effective bathymetry. Still, our results demonstrate that even in the supercritical regime, linear theory can explain the lee wave drag. In this sense, they represent a qualified triumph of linear lee wave theory.

Acknowledgments. We gratefully acknowledge the support of ONR Grant N00014-16-1-2256 (scientific officers Dr. T. Paluszkiewicz and Dr. S. Harper).

Data availability statement. MATLAB data files for each simulation as well as MATLAB scripts to generate all figures used in this paper are provided in the Stanford Digital Repository (https://purl.stanford.edu/jy494jz4178).

\section{REFERENCES}

Bell, T. H., 1975a: Statistical features of sea-floor topography. Deep-Sea Res. Oceanogr. Abstr., 22, 883-892, https://doi.org/ 10.1016/0011-7471(75)90090-X.

- 1975b: Topographically generated internal waves in the open ocean. J. Geophys. Res., 80, 320-327, https://doi.org/10.1029/ JC080i003p00320.

Cusack, J. M., A. C. Naveira Garabato, D. A. Smeed, and J. B. Girton, 2017: Observation of a large lee wave in the Drake Passage. J. Phys. Oceanogr., 47, 793-810, https://doi.org/ 10.1175/JPO-D-16-0153.1.

Eckermann, S. D., J. Lindeman, D. Broutman, J. Ma, and Z. Boybeyi, 2010: Momentum fluxes of gravity waves generated by variable Froude number flow over three-dimensional obstacles. J. Atmos. Sci., 67, 2260-2278, https://doi.org/10.1175/2010JAS3375.1.

Ferrari, R., and C. Wunsch, 2009: Ocean circulation kinetic energy: Reservoirs, sources, and sinks. Annu. Rev. Fluid Mech., 41, 253-282, https://doi.org/10.1146/annurev.fluid.40.111406.102139.

Fringer, O. B., M. Gerritsen, and R. L. Street, 2006: An unstructured-grid, finite-volume, nonhydrostatic, parallel coastal ocean simulator. Ocean Modell., 14, 139-173, https:// doi.org/10.1016/j.ocemod.2006.03.006. 
Garner, S. T., 2005: A topographic drag closure built on an analytical base flux. J. Atmos. Sci., 62, 2302-2315, https://doi.org/ 10.1175/JAS3496.1.

Gill, A. E., 1982: Atmosphere-Ocean Dynamics. Academic Press, $662 \mathrm{pp}$.

Goff, J. A., and T. H. Jordan, 1988: Stochastic modeling of seafloor morphology: Inversion of sea beam data for second-order statistics. J. Geophys. Res., 93, 13 589-13 608, https://doi.org/ 10.1029/JB093iB11p13589.

—, and B. K. Arbic, 2010: Global prediction of abyssal hill roughness statistics for use in ocean models from digital maps of paleo-spreading rate, paleo-ridge orientation, and sediment thickness. Ocean Modell., 32, 36-43, https://doi.org/10.1016/ j.ocemod.2009.10.001

Klymak, J. M., 2018: Nonpropagating form drag and turbulence due to stratified flow over large-scale abyssal hill topography. J. Phys. Oceanogr., 48, 2383-2395, https://doi.org/10.1175/JPO-D-17-0225.1.

Kunze, E., and R. Lien, 2019: Energy sinks for lee waves in shear flow. J. Phys. Oceanogr., 49, 2851-2865, https://doi.org/10.1175/ JPO-D-19-0052.1.

Lilly, D. K., and J. B. Klemp, 1979: The effects of terrain shape on nonlinear hydrostatic mountain waves. J. Fluid Mech., 95, 241261, https://doi.org/10.1017/S0022112079001452.

Lott, F., and M. J. Miller, 1997: A new subgrid-scale orographic drag parametrization: Its formulation and testing. Quart. J. Roy. Meteor. Soc., 123, 101-127, https://doi.org/10.1002/ qj. 49712353704 .

Mayer, F. T., and O. B. Fringer, 2017: An unambiguous definition of the Froude number for lee waves in the deep ocean. J. Fluid Mech., 831, R3, https://doi.org/10.1017/JFM.2017.701.

Melet, A., R. Hallberg, A. Adcroft, M. Nikurashin, and S. Legg, 2015: Energy flux into internal lee waves: Sensitivity to future climate changes using linear theory and a climate model. J. Climate, 28 2365-2384, https://doi.org/10.1175/JCLI-D-14-00432.1.

Naveira Garabato, A. C., A. J. G. Nurser, R. B. Scott, and J. A. Goff, 2013: The impact of small-scale topography on the dynamical balance of the ocean. J. Phys. Oceanogr., 43, 647-668, https://doi.org/10.1175/JPO-D-12-056.1.

Nelson, K. S., and O. B. Fringer, 2017: Reducing spin-up time for simulations of turbulent channel flow. Phys. Fluids, 29, 105101 https://doi.org/10.1063/1.4993489.

Nikurashin, M., and R. Ferrari, 2010: Radiation and dissipation of internal waves generated by geostrophic motions impinging on small-scale topography: Theory. J. Phys. Oceanogr., 40, 10551074, https://doi.org/10.1175/2009JPO4199.1.

— and _-, 2011: Global energy conversion rate from geostrophic flows into internal lee waves in the deep ocean. Geophys. Res. Lett., 38, L08610, https://doi.org/10.1029/2011GL046576.

,,-- N. Grisouard, and K. Polzin, 2014: The impact of finiteamplitude bottom topography on internal wave generation in the Southern Ocean. J. Phys. Oceanogr., 44, 2938-2950, https://doi.org/10.1175/JPO-D-13-0201.1.

Palmer, T. N., G. J. Shutts, and R. Swinbank, 1986: Alleviation of a systematic westerly bias in general circulation and numerical weather prediction models through an orographic gravity wave drag parametrization. Quart. J. Roy. Meteor. Soc., 112 1001-1039, https://doi.org/10.1002/qj.49711247406.

Pedlosky, J., 2003: Waves in the Ocean and Atmosphere: Introduction to Wave Dynamics. Springer, $260 \mathrm{pp}$.

Peltier, W. R., and T. L. Clark, 1979: The evolution and stability of finite-amplitude mountain waves. Part II: Surface wave drag and severe downslope windstorms. J. Atmos. Sci., 36,
1498-1529, https://doi.org/10.1175/1520-0469(1979)036<1498: TEASOF $>2.0 . \mathrm{CO} ; 2$

Pierrehumbert, R., 1987: An essay on the parameterization of orographic gravity wave drag. Seminar/Workshop on Observation, Theory and Modelling of Orographic effects, Reading, United Kingdom, ECMWF, 251-282, https://www.ecmwf.int/node/11673.

Scott, R. B., J. A. Goff, A. C. Naveira Garabato, and A. J. G. Nurser, 2011: Global rate and spectral characteristics of internal gravity wave generation by geostrophic flow over topography. J. Geophys. Res., 116, C09029, https://doi.org/10.1029/2011JC007005.

Shakespeare, C. J., 2020: Interdependence of internal tide and lee wave generation at abyssal hills: Global calculations. J. Phys. Oceanogr., 50, 655-677, https://doi.org/10.1175/JPO-D-19-0179.1. , and A. M. Hogg, 2017: The viscous lee wave problem and its implications for ocean modelling. Ocean Modell., 113, 22-29, https://doi.org/10.1016/j.ocemod.2017.03.006.

Stein, J., 1992: Investigation of the regime diagram of hydrostatic flow over a mountain with a primitive equation model. Part I: Twodimensional flows. Mon. Wea. Rev., 120, 2962-2976, https:// doi.org/10.1175/1520-0493(1992)120<2962:IOTRDO>2.0.CO;2.

St. Laurent, L. C., H. L. Simmons, and S. R. Jayne, 2002: Estimating tidally driven mixing in the deep ocean. Geophys. Res. Lett., 29, 2106, https://doi.org/10.1029/2002GL015633.

Thyng, K. M., C. A. Greene, R. D. Hetland, H. M. Zimmerle, and S. F. DiMarco, 2016: True colors of oceanography: Guidelines for effective and accurate colormap selection. Oceanography, 29, 9-13, https://doi.org/10.5670/oceanog.2016.66.

Trossman, D. S., and Coauthors, 2013: Impact of parameterized lee wave drag on the energy budget of an eddying global ocean model. Ocean Modell., 72, 119-142, https://doi.org/10.1016/j.ocemod.2013.08.006. and Coauthors, 2015: Internal lee wave closures: Parameter sensitivity and comparison to observations. J. Geophys. Res. Oceans, 120, 7997-8019, https://doi.org/10.1002/2015JC010892.

_, B. K. Arbic, J. G. Richman, S. T. Garner, S. R. Jayne, and A. J. Wallcraft, 2016: Impact of topographic internal lee wave drag on an eddying global ocean model. Ocean Modell., 97, 109-128, https://doi.org/10.1016/j.ocemod.2015.10.013.

Waterman, S., A. C. Naveira Garabato, and K. L. Polzin, 2013: Internal waves and turbulence in the Antarctic circumpolar current. J. Phys. Oceanogr., 43, 259-282, https://doi.org/10.1175/JPO-D-11-0194.1.

Welch, W. T., P. Smolarkiewicz, R. Rotunno, and B. A. Boville, 2001: The large-scale effects of flow over periodic mesoscale topography. J. Atmos. Sci., 58, 1477-1492, https://doi.org/ 10.1175/1520-0469(2001)058<1477:TLSEOF $>2.0$. CO;2.

Winters, K. B., 2016: The turbulent transition of a supercritical downslope flow: Sensitivity to downstream conditions. J. Fluid Mech., 792, 997-1012, https://doi.org/10.1017/jfm.2016.113.

Wright, C. J., R. B. Scott, P. Ailliot, and D. Furnival, 2014: Lee wave generation rates in the deep ocean. Geophys. Res. Lett., 41, 2434-2440, https://doi.org/10.1002/2013GL059087.

Yang, L., M. Nikurashin, A. M. Hogg, and B. M. Sloyan, 2018: Energy loss from transient eddies due to lee wave generation in the Southern Ocean. J. Phys. Oceanogr., 48, 2867-2885, https://doi.org/10.1175/JPO-D-18-0077.1.

Yang, Q., M. Nikurashin, H. Sasaki, H. Sun, and J. Tian, 2019: Dissipation of mesoscale eddies and its contribution to mixing in the northern South China Sea. Sci. Rep., 9, 556, https:// doi.org/10.1038/S41598-018-36610-X

Zheng, Q., B. Holt, X. Li, X. Liu, Q. Zhao, Y. Yuan, and X. Yang, 2012: Deep-water seamount wakes on SEASAT SAR image in the Gulf Stream region. Geophys. Res. Lett., 39, L16604, https://doi.org/10.1029/2012gl052661. 